\title{
Growth and Aggregation Regulate Clusters Structural Properties and Gel Time
}

\author{
Stefano Lazzari*, ${ }^{\dagger}$ and Marco Lattuada ${ }^{*} \neq$ \\ ${ }^{\dagger}$ Department of Chemical Engineering, Massachusetts Institute of Technology, 77, Massachusetts Avenue, Cambridge, Massachusetts \\ 02139, United States \\ ${ }^{\ddagger}$ Department of Chemistry, Université de Fribourg, Chemin du Musée 9, 1700 Fribourg, Switzerland
}

\section{Supporting Information}

\begin{abstract}
When particles undergo aggregation, very often they form structures that can be described with fractal geometry concepts. The spatial organization of the particles embedded in these aggregates, quantified by means of their fractal dimension, plays a key role in the clusters' diffusion and aggregation process. Fractal dimensions are typically known for some specific, ideal aggregation scenarios, such as for diluted diffusion limited cluster aggregation (DLCA) or reaction limited cluster aggregation (RLCA). The situation becomes significantly more complicated as soon as the initial particle concentration increases and fractal-dimension changing phenomena, such as particle growth, occur. In this frame, the aim of the present work is twofold: (i) to investigate the clusters' spatial organization in a scenario where growth and aggregation occur simultaneously and (ii) to assess the corresponding aggregation kinetics. To this end, an ad hoc Monte Carlo

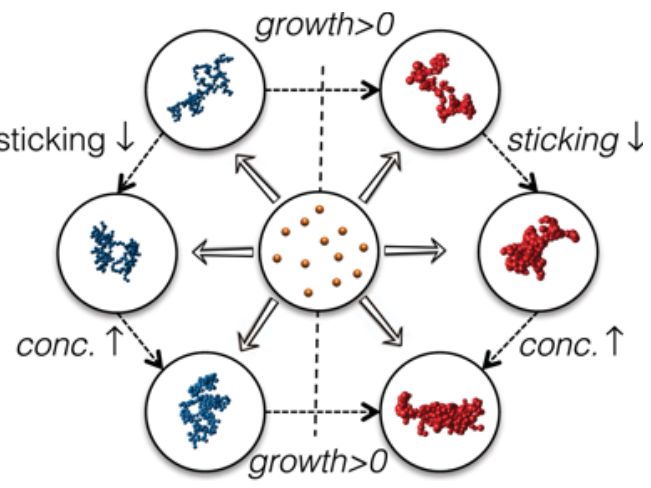
model has been developed. Both DLCA and RLCA regimes have been explored at several initial primary particle concentrations and for different growth rates. The results were discussed in terms of the characteristic times of growth $\left(\tau_{\mathrm{G}}\right)$ and aggregation $\left(\tau_{\mathrm{A}}\right)$, as well as the rate at which the structural properties change, $v_{\mathrm{R}}$. It was then possible to propose empirical correlations in the form $d_{\mathrm{m}}=d_{\mathrm{m}}\left(v_{\mathrm{R}}\right)$ and $t_{\text {gel }}=t_{\text {gel }}\left(\tau_{\mathrm{A}}, \tau_{\mathrm{G}}\right)$ to relate the evolution of the mass mobility exponent $d_{\mathrm{m}}$ and the gel times to the simultaneously occurring processes of growth and aggregation.
\end{abstract}

\section{INTRODUCTION}

The formation and growth of crystals in suspensions are central in many applications, ranging from imaging to photovoltaics, to drug formulation. ${ }^{1,2}$ For a crystal-based product to be useful, effective, and safe, characteristics such as crystal size, shape, and polymorphism have to be well controlled. ${ }^{3-5}$ In the past few decades, experimental and modeling investigations have clarified how to tune the aforementioned properties relying on variables such as temperature, solvents, surface tension, and supersaturation. ${ }^{3,5-7}$ The gathered knowledge allowed the development of deterministic models, describing the timeevolution of the crystal size distributions, opening up the possibility to better control and optimize their production processes. ${ }^{1,8,9}$

Typically, when modeling crystal formation, a complex number of phenomena need to be included, such as crystal nucleation, growth, agglomeration, and breakage. ${ }^{1,10}$ As a result of the agglomeration of particles, clusters with highly irregular structures are often produced. The geometrical features of these clusters cannot be described by means of conventional Euclidean geometry. Thanks to the pioneering work of Mandelbrot, it was discovered that fractal geometry concepts could be applied to the description of particle agglomerates. The fractal dimension, in particular, is the parameter defining the compactness of clusters. To describe the kinetics of agglomeration and breakage using a population balance equations framework, the fractal dimension $\left(d_{\mathrm{f}}\right)$ of the clusters is typically required, as it appears in the kinetic expressions describing these events. ${ }^{11,12}$ The fractal dimension describes the spatial organization of the clusters and is typically between one (linear clusters) and three (homogeneous assemblies). ${ }^{12}$ Note that in the following discussion, the terms aggregation and agglomeration will be employed to describe the clustering process of primary particles. Aggregation refers to clusters held together by van der Waals forces, and agglomeration refers to structures whose particles are cemented by crystalline bridges. In the most general sense, $d_{\mathrm{f}}$ is a function of the aggregation regime considered. For instance, in diffusion limited cluster aggregation (DLCA) $d_{\mathrm{f}}=1.8$, whereas larger values of about $d_{\mathrm{f}}$ $=2.1$ have been reported in reaction limited cluster aggregation (RLCA). ${ }^{13}$ As has been recently pointed out, however, discrepancies arise from these values when the colloidal system undergoes $d_{\mathrm{f}}$ changing phenomena whilst aggregation or breakage occur. ${ }^{12}$ For instance, if restructuring occurs during aggregation, significant deviations in fractal dimensions are observed both experimentally, as well as computationally. ${ }^{14,15} \mathrm{~A}$ 
further example is aggregating particles that simultaneously coalesce. In this case, the fractal dimension shifts toward larger values, as compared to that of the noncoalescing cases. ${ }^{16}$ It has been shown that for both aerosols and polymeric particles the ratio of aggregation and coalescence characteristic times regulates the fractal dimension and therefore the aggregation process. ${ }^{16,17}$ A similar reasoning can be applied to systems where agglomeration and growth occur simultaneously, such as crystals. If growth occurs at a much larger rate as compared to that of agglomeration, crystals will become compact, with a $d_{\mathrm{f}} \approx$ 3. Conversely, when the growth process is slow compared to that of agglomeration events, fractal-like crystals with $d_{\mathrm{f}} \approx 2$ are observed. These two limiting cases have been shown to be strongly dependent on the supersaturation $(S)$, that is, the ratio between the available solute concentration and the solubility limit of the same substance. ${ }^{18}$ Supersaturation is strongly connected to the growth process; the larger the $S$, the faster the growth rate of crystals. ${ }^{3,18}$ In fact, it was shown that at higher supersaturation ratios, one obtains a faster growth and more compact crystals, as compared to lower supersaturation, which leads to open structures. ${ }^{18}$

As opposed to growth models, no universally accepted mathematical framework exists to describe crystal agglomeration; therefore, the employed kernels remain empirical in nature. ${ }^{19}$ To attempt a more fundamental description, knowledge of the $d_{\mathrm{f}}$ of growing and agglomerating clusters would be beneficial. Most crystallization processes occur at high values of supersaturation, and the $d_{\mathrm{f}}$ of the crystals may be assumed to be equal to 3 , given that crystals grow quickly at high $S$, and potential voids of the forming clusters are rapidly filled. Although this condition is often fulfilled, situations exist where agglomeration prevails over, or at least competes with, growth, for example, in the undesired formation of crystals in flow reactors, often resulting in clogging. ${ }^{20}$ When crystals are the undesired side-product of a reaction, their supersaturation might be low, resulting in ramified crystals, which, after nucleating and growing, may deposit in the reactor and clog it upon agglomeration, as has been shown in the case of amination and cross-coupling reactions. ${ }^{20,21}$ Moreover, the interplay of growth and agglomeration is relevant in the formation of inorganic nanocrystals (e.g., gold, silver, iron, or silica-based particles). Although such particles are being synthesized with quite reasonable control over their size and polydispersity, their formation mechanism has not been yet unraveled. ${ }^{22}$ Moreover, several interesting structures consisting of inorganic nanocrystals have significant application potential in the fields of catalytic supports and insulators. ${ }^{23,12}$

Different methodologies, relying on either experimental and/ or modeling approaches allow the determination of the fractal dimension. ${ }^{12,24}$ Among the modeling approaches, the most successful technique in this sense, is the Monte Carlo (MC) simulation. ${ }^{11,25,26}$ Such a methodology allows simulation of a predefined number of particles moving in a box and agglomerating with one another according to a given set of rules (e.g., particles stick upon contact, mimicking a DLCA case). During the simulation, the clustering process is followed, tracking both the size of the clusters and the positions of the particles that comprise them. This allows calculation of the hydrodynamic radii of any $i$-sized cluster. Although this is, in general, a highly complex task, approximate approaches, such as the Kirkwood-Rieseman method, permit estimation of $R_{\mathrm{H}, i}$ if all primary particles have the same size, as
$\frac{N_{\mathrm{p}} R_{\mathrm{p}, 0}}{R_{\mathrm{H}, i}}=1+\frac{1}{N_{\mathrm{p}}^{2}} \sum_{i \neq j} \frac{1}{r_{i j}}$, where $N_{\mathrm{p}}$ is the number of particles in a cluster, $R_{\mathrm{p}, 0}$ is the primary particle radius, and $r_{i j}$ is the distance between the centers of particles $i$ and $j$. From $R_{\mathrm{H}, i}$, the so-called mass mobility exponent, $d_{\mathrm{m}}$ can be obtained from the following expression, defining the fractal scaling of the cluster

$$
i=k\left(\frac{R_{\mathrm{H}, i}}{R_{\mathrm{p}, 0}}\right)^{d_{\mathrm{m}}}
$$

where $i$ is the cluster mass (i.e., the number of particles constituting a cluster) and $k$ is the fractal prefactor. Note that $d_{\mathrm{m}}$ is strictly related to $d_{\mathrm{f}}$, as both quantities reflect the structural properties of colloidal clusters. The main difference is that $d_{\mathrm{f}}$ is computed from the gyration radius, and $d_{\mathrm{m}}$ from the hydrodynamic radius. This methodology has been validated and successfully applied for more than 30 years. ${ }^{1,24,25,27-29}$

In this frame, a MC model able to describe the simultaneous growth and aggregation of particles has been developed to (i) evaluate the $d_{\mathrm{m}}$ of the growing/aggregating particles in different conditions and (ii) assess the corresponding kinetics. In particular, a growth mechanism has been implemented in a MC code able to describe DLCA and RLCA. ${ }^{27,28}$ The clusters' mass-mobility exponent is evaluated as a function of parameters such as the growth rate, the initial occupied volume fraction, and the particles sticking probability. The present model represents an interesting tool able to provide the kinetic evolution, as well as the fractal properties, of clusters undergoing simultaneous growth and aggregation/agglomeration processes.

\section{MC SIMULATIONS}

2.1. The MC Algorithm. The employed MC model has been developed starting from a previously validated model. ${ }^{27,28}$ The main difference is the existence of a growth step. In particular, the MC algorithm proceeds as follows:

(i) a number $N$ of equally sized particles (radius $R_{\mathrm{p}, 0}$ ) are randomly placed in a box of size $L$ avoiding superimpositions, once the desired initial occupied volume fraction $\phi_{0}$ is selected

$$
\phi_{0}=\frac{4}{3} \pi R_{\mathrm{p}, 0}^{3} \frac{N}{L^{3}}
$$

(ii) at every iteration, a particle (or a cluster) is randomly selected with a probability $P_{\mathrm{D}, i}$, proportional to its diffusion coefficient $D_{\text {iff,i } i}$ which is a function of the hydrodynamic radius $R_{\mathrm{H}, i}$, according to the StokesEinstein law ${ }^{11}$

$$
D_{\mathrm{iff}, i}=\frac{k_{\mathrm{B}} T}{6 \pi \eta R_{\mathrm{H}, i}}
$$

where $k_{\mathrm{B}}$ is the Boltzmann constant, $T$ is the temperature, and $\eta$ is the viscosity of the continuous phase. The resulting probability $P_{\mathrm{D}, i}$ that a cluster is selected is then

$$
P_{\mathrm{D}, i}=\frac{D_{\mathrm{iff}, i}}{\sum_{i=1}^{N_{\mathrm{c}}} D_{\mathrm{iff}, i}}=\frac{R_{\mathrm{H}, i}^{-1}}{\sum_{i=1}^{N_{\mathrm{c}}} R_{\mathrm{H}, i}^{-1}}
$$

where $N_{\mathrm{C}}$ is the total number of clusters in the box. The randomly selected cluster is moved by a fraction $\alpha$ of the initial primary particle radius. In the case of a collision between two 
particles (or clusters), a new cluster is formed with a given sticking probability, $P_{\mathrm{S}}$. In the DLCA case, $P_{\mathrm{S}}=1$, whereas $P_{\mathrm{S}}$ $\ll 1$ under RLCA conditions. In the case of RLCA, whenever a collision is ineffective, the clusters do not fully bounce back, but are moved by $80 \%$ of the distance necessary to have a collision;

(iii) at every iteration, a random particle is selected (hence with probability $1 / N$ ) and its dimension is increased by a quantity $\Delta R$, which is proportional to a fraction of the initial particle radius, $g_{\mathrm{R}}$, corrected by a factor corresponding to the length of the time step as will be detailed later on;

(iv) every time a new cluster is formed, or a particle grows, the corresponding hydrodynamic radius is recalculated, as well as the volume and the surface area of the aggregate. The two latter quantities are calculated using the ARVO package, which was integrated into the MC code $^{30}$ All of these quantities account for the particles' partial superimposition;

(v) the simulation ends either when either (a) a maximum number of iterations is reached, (b) only a few clusters are left in the box (e.g., 20), or (c) a "super-cluster" (the so-called gel) arises, spanning the whole box, connecting all of its surfaces.

Note that when two particles are adjacent in a cluster, if one grows substantially, it will incorporate the other one. The growth of the particles is assumed to be constant throughout the simulation, implying a constant supersaturation, a condition that has also been explored experimentally. ${ }^{31,32}$ Moreover, all particles possess the same growth rate, whatever their size. Although these assumptions can be straightforwardly changed in the code, they have been made for the sake of simplicity, as this work represents the very first attempt to describe simultaneous growth and aggregation with a MC code. A further point worth mentioning is that the initial particle radii, $R_{\mathrm{p}, 0}$, is equal to $\sqrt{ } 3 / 2 L$. Therefore, eq 2 allows calculation of the size of the employed box, once the occupied volume fraction $\phi_{0}$ is selected.

From the description of the MC algorithm, it is clear that the calculation of the cluster hydrodynamic radius is of crucial importance. Because clusters are comprised of partially overlapping spheres with different radii, due to the growth process, the calculation of the cluster hydrodynamic radius has been carried out using an extension of the method presented in a previous work. ${ }^{17}$ The details are presented in the electronic Supporting Information.

2.2. MC Time and Physical Time. During a MC simulation, the time increment at each step is given by the time a particle requires to diffuse a distance equal to its diameter (or a fraction $\alpha$ thereof), divided by the total number of clusters present, $N_{\mathrm{C}}$

$$
\Delta t_{\mathrm{MC}}=\frac{\alpha^{2}}{\sum_{i=1}^{N_{\mathrm{C}}} \frac{R_{\mathrm{P}, 0}}{R_{\mathrm{H}, i}}}
$$

Note that all radii are normalized by $R_{\mathrm{P}, 0}$. To link the MC time, $\Delta t_{\mathrm{MC}}$, to the physical time, one needs to multiply it with the time $\tau_{\mathrm{D}}$ for a particle to diffuse by a quantity corresponding to its diameter (cf. eq 3 )

$$
\Delta t=\Delta t_{\mathrm{MC}} \tau_{\mathrm{D}}=\frac{\alpha^{2}}{\sum_{i=1}^{N_{\mathrm{C}}} \frac{R_{\mathrm{P}, 0}}{R_{\mathrm{H}, i}}} \cdot \frac{\left(2 R_{\mathrm{P}, 0}\right)^{2}}{6 D_{\mathrm{iff}, 1}}=\frac{\alpha^{2}}{\sum_{i=1}^{N_{\mathrm{C}}} \frac{R_{\mathrm{P}, 0}}{R_{\mathrm{H}, i}}} \cdot \frac{4 \pi \eta R_{\mathrm{P}, 0}^{3}}{k_{\mathrm{B}} T}
$$

When describing the $\mathrm{MC}$ algorithm, in step (iii), a timecorrection for the radius growth rate $\Delta R$, was mentioned. This is described by

$$
\Delta R=R_{\mathrm{P}, 0} g_{\mathrm{R}} N \Delta t_{\mathrm{MC}}
$$

Note that $N$ is the number of particles in the system. Multiplying by $N$ implies that at the beginning of the simulation, where $\Delta t_{\mathrm{MC}}=1 / N$ for $\alpha=1$ (cf. eq 5), $\Delta R=$ $R_{\mathrm{P}, 0} g_{\mathrm{R}}$. Hence, the growth of a primary particle is proportional to a fraction $g_{\mathrm{R}}$ of its radius $R_{\mathrm{P}, 0}$. Having already linked the MC time $t_{\mathrm{MC}}$ to the physical time $t$ (cf. eq 6), it is possible to compute the physical growth time. Note that $\alpha=1$ has been used in all of our simulations, which is consistent with previously validated MC models. ${ }^{27,28}$

\section{RESULTS AND DISCUSSION}

Before illustrating the results, it is worth introducing some useful quantities, which represent the features of the cluster mass distribution obtained from the MC simulations. In particular, given the cluster mass distribution $N_{i}$, it is possible to calculate the $k$-th moments of the distribution, $\mu_{k}$, and the average occupied volume fraction $\phi$

$$
\begin{aligned}
& \mu_{k}=\sum_{i=1}^{N_{\mathrm{C}}}{ }^{k} N_{i} \\
& \phi=\frac{4}{3} \pi \frac{1}{L^{3}} \sum_{i=1}^{N_{\mathrm{C}}} N_{i} R_{\mathrm{H}, i}^{3}
\end{aligned}
$$

To extract the $d_{\mathrm{m}}$ and the fractal prefactor $k$, eq 1 is employed. At different times, the cluster mass distribution is reported on a $\log -\log$ plot in terms of $i$ versus $R_{\mathrm{H}, i}$. The resulting slope and intercept represent $d_{\mathrm{m}}$ and $k$, respectively. Further details, in this sense, are found in the Supporting Information, in particular Figure S1.

A further useful tool to discuss the simulation results is the characteristic time of aggregation, $\tau_{\mathrm{A}}$, and growth $\tau_{\mathrm{G}}$

$$
\begin{aligned}
\tau_{\mathrm{A}} & =\frac{1}{\beta_{11} C_{\mathrm{P}, 0}} \\
\tau_{\mathrm{G}} & =\frac{1}{k_{\mathrm{G}}}
\end{aligned}
$$

where $k_{\mathrm{G}}\left(\mathrm{s}^{-1}\right)$ is the rate of growth, $C_{\mathrm{P}, 0}$ is the primary particle concentration at time zero, and $\beta_{11}$ is the aggregation rate between two primary particles

$$
\beta_{11}=\frac{8}{3} \frac{k_{\mathrm{B}} T}{W \eta}
$$

Note that $W$ is the Fuchs stability factor, equal to 1 in DLCA and larger than 1 in the RLCA case. These characteristic times need to be redefined in the frame of the MC simulations, given that the aggregation and growth processes are linked to probabilities, particle numbers, and their mobility in the box, and indirectly comprising more physical parameters (e.g., temperature, viscosity) 


$$
\begin{aligned}
\tau_{\mathrm{A}} & =\frac{1}{\beta_{11} C_{\mathrm{P}, 0}}=\underbrace{\frac{3 \eta V_{p, 0}}{8 k_{\mathrm{B}} T}}_{\varepsilon} \frac{W}{\phi_{0}}=\varepsilon \frac{1}{P_{\mathrm{S}} \phi_{0}} \\
\tau_{\mathrm{G}} & =\frac{1}{k_{\mathrm{G}}} \propto \frac{1}{g_{\mathrm{R}}}
\end{aligned}
$$

where $\phi_{0}$ is the occupied volume fraction at time zero (cf. eq 2), $V_{\mathrm{p}, 0}$ is the primary particle volume at time zero, and $C_{\mathrm{p}, 0}=$ $\phi_{0} / V_{\mathrm{p}, 0}$. $P_{\mathrm{S}}$ is the sticking probability, assumed to be equal for any $i$-sized cluster, and $g_{\mathrm{R}}$ is the aforementioned \% growth rate increase of a primary particle. It has been assumed that $W=P_{\mathrm{S}}^{-1}$ and that $k_{\mathrm{G}} \propto g_{\mathrm{R}}$. Note that for $g_{\mathrm{R}} \rightarrow 0$ the characteristic time of growth becomes infinite. Defining the ratio between the aggregation and growth characteristic times, $\psi$, one has

$$
\psi=\frac{\tau_{\mathrm{A}}}{\tau_{\mathrm{G}}} \propto \frac{g_{\mathrm{R}}}{P_{\mathrm{S}} \phi_{0}}
$$

The ratio $\psi$ can take values between 0 (no growth occurs) and potentially infinity, for very small amounts of particles or a very small sticking probability. $\psi$ offers the possibility to combine the relevant parameters in the interplay of aggregation and growth, and to assess their relative importance.

As a matter of fact, the parameter values employed in the DLCA and RLCA simulations (reported in Table 1) have been varied to explore values of $\psi$ between $10^{-2}$ and $10^{1}$.

Table 1. Parameter Values Employed in the Simulations

\begin{tabular}{lcccc} 
& $N(-)$ & $\phi_{0}(\%)$ & $P_{\mathrm{S}}(-)$ & $g_{\mathrm{R}}(\%)$ \\
DLCA & $5 \times 10^{4}$ & $1 / 2 / 5 / 10$ & 1 & $0.00 / 0.25 / 0.50 / 0.75 / 1.00$ \\
RLCA & $5 \times 10^{4}$ & $1 / 2 / 5 / 10$ & 0.1 & $0.00 / 0.25 / 0.50 / 0.75 / 1.00$ \\
\hline
\end{tabular}

Note that the employed box size $L$ changes to accommodate different initial occupied volume fractions $\phi_{0}$, whilst keeping a constant number of particles $N$ (cf. eq 2). In particular, $L=239$,
190,140 , and 111 when $\phi_{0}$ was equal to $1,2,5$, and $10 \%$, respectively. All simulation results reported were averaged over 100 repetitions. The length of each simulation increases with growth rate, as particle overlapping has to be accounted for. Running 100 repetitions with our nonparallelized code required about $4 \mathrm{~h}$ on a 12 core machine with $2.40 \mathrm{GHz}$ CPU.

Note that the cluster masses used to compute the $d_{\mathrm{m}}$ fall between two values, $i_{\mathrm{MIN}}$ and $i_{\mathrm{MAX}} \cdot i_{\mathrm{MIN}}=10$ was selected as suggested in the literature, ${ }^{33}$ whereas $i_{\mathrm{MAX}}=5000$ was selected through numerical experiments (cf. Figure S1) to avoid biases due to the selected box size in which the particles "move". All other average properties reported (e.g., moments and occupied volume fractions) were computed considering the whole cluster mass distribution. To avoid further "box effects" the simulations were considered only until the gel time, that is, the moment where a "super-cluster" spans the box, connecting all of its sides.

To properly compare the simulation results, which take place on different time scales given the range of $\phi_{0}$ explored, a normalized time, $\tau_{\mathrm{N}}$ is employed

$$
\tau_{\mathrm{N}}=\frac{t}{\tau_{\mathrm{A}}}
$$

Recalling that $t=t_{\mathrm{MC}} \tau_{\mathrm{D}}$ (cf. eq 6$), W=P_{\mathrm{S}}^{-1}$, and using eq 13 one gets

$$
\tau_{\mathrm{N}}=t_{\mathrm{MC}} \frac{\tau_{\mathrm{D}}}{\tau_{\mathrm{A}}}=t_{\mathrm{MC}} \underbrace{\frac{4 \pi \eta R_{\mathrm{P}, 0}^{3}}{k_{\mathrm{B}} T}}_{\tau_{\mathrm{D}}} \underbrace{\frac{8 k_{\mathrm{B}} T \phi_{0}}{3 \eta V_{\mathrm{p}, 0} W}}_{\tau_{\mathrm{A}}^{-1}}=t_{\mathrm{MC}} 8 \phi_{0} P_{\mathrm{S}}
$$

A further advantage of discussing the simulation results as a function of $\tau_{\mathrm{N}}$ is that such a characteristic time is often used to rationalize experimental results in the frame of colloid aggregation. $^{17}$

3.1. DLCA. In the DLCA regime, clusters stick upon contact; therefore, the sole parameters affecting the cluster

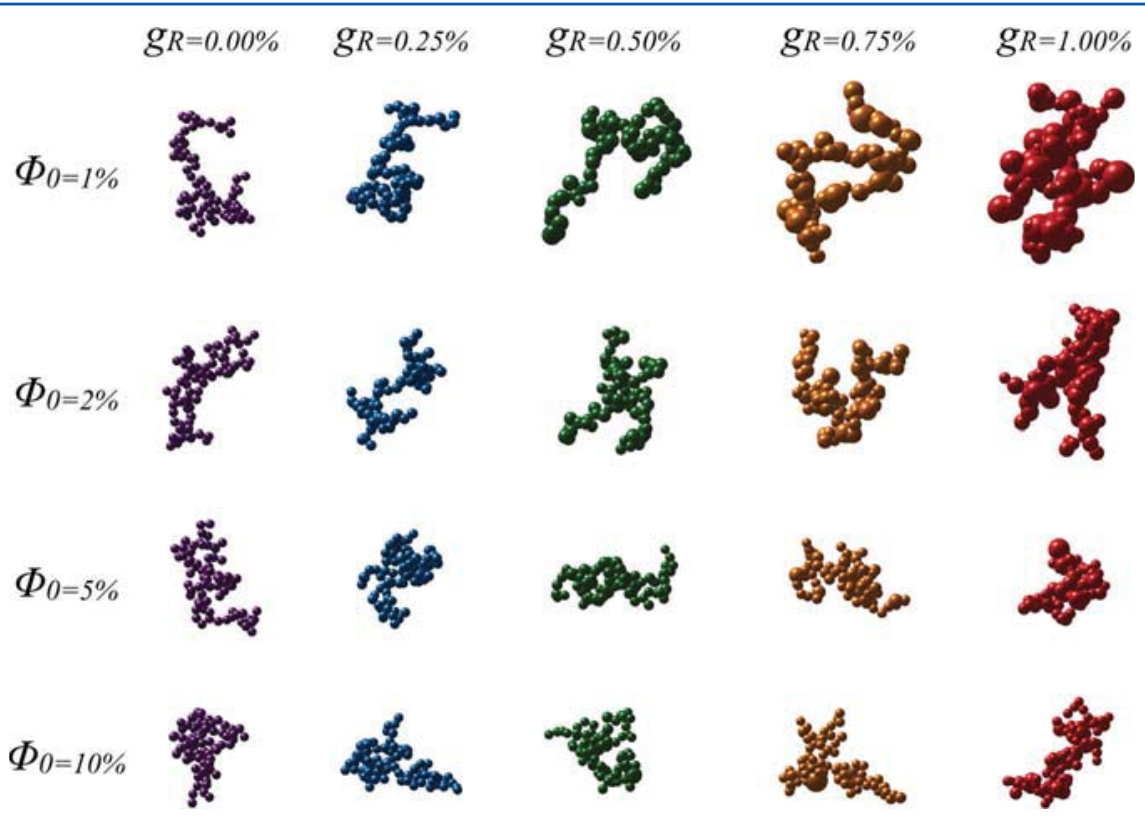

Figure 1. Examples of DLCA clusters retrieved at $\tau_{\mathrm{N}}=10$, with mass $i$ between $70<i<90$. Clusters are generated at different initial occupied volume fractions $\left(\phi_{0}=1,2,5\right.$, and $\left.10 \%\right)$ and growth rates $g_{\mathrm{R}}$. Color code: violet, $g_{\mathrm{R}}=0.00 \%$; blue, $g_{\mathrm{R}}=0.25 \%$; green, $g_{\mathrm{R}}=0.50 \%$; orange, $g_{\mathrm{R}}=$ $0.75 \%$; red, $g_{\mathrm{R}}=1.00 \%$. 

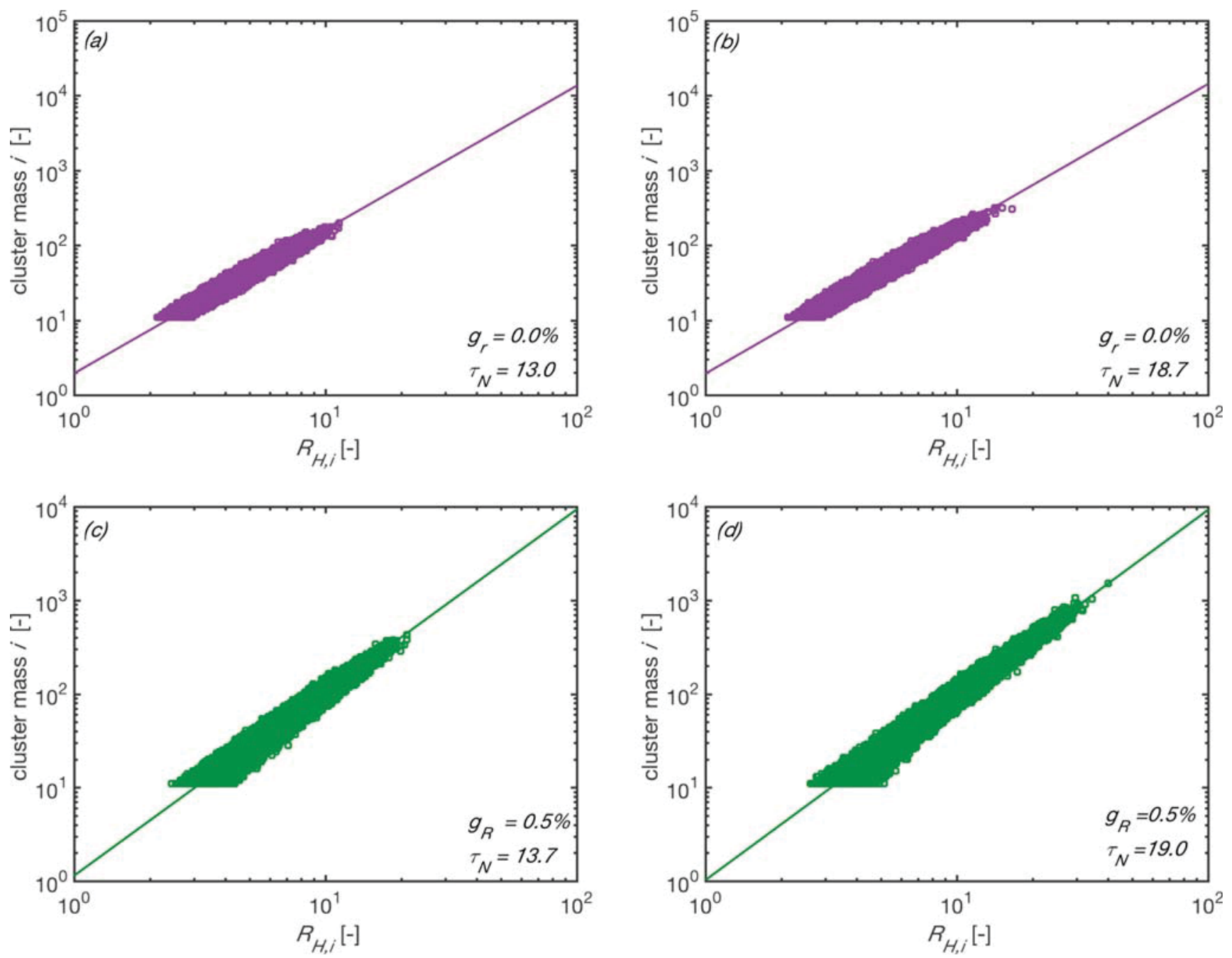

Figure 2. $R_{\mathrm{H}, i}$ against cluster mass $i$ at different $\tau_{\mathrm{N}}$ for the DLCA case at $\phi_{0}=1 \%$ with $g_{\mathrm{R}}=0.00 \%(\mathrm{a}, \mathrm{b})$ and with $g_{\mathrm{R}}=0.50 \%(\mathrm{c}, \mathrm{d})$. The reported cluster masses fall between $i_{\mathrm{MIN}}=10$ and $i_{\mathrm{MAX}}=5000$. The squares represent $R_{\mathrm{H}, i}$ at a given cluster mass $i$, and the continuous lines are obtained using the equation $R_{\mathrm{H}, i}=R_{\mathrm{p}}(i / k)^{\left(1 / d_{\mathrm{m}}\right)}$, where $k$ and $d_{\mathrm{m}}$ are fitted against the reported data points.

structure are the growth rate, $g_{\mathrm{R}}$ and the initial occupied volume fraction, $\phi_{0}$. To qualitatively appreciate the impact of these quantities on the cluster structures formed, aggregates of a comparable mass $(70<i<90)$ extracted at the same time $\tau_{\mathrm{N}}$ $=10$ are reported in Figure 1 .

Two effects can be appreciated when inspecting Figure 1. When $\phi_{0}$ increases, more compact clusters are observed. Although this effect is observed at any value of $g_{\mathrm{R}}$, it is most evident when considering the nongrowing clusters, with $g_{R}=$ $0.00 \%$ (cf. violet clusters in Figure 1). This result is expected as the higher particle concentration progressively drives the system away from the ideal DLCA conditions, where more open clusters are formed. ${ }^{11,12,33,34}$ In terms of characteristic times, compact clusters arise for smaller $\tau_{\mathrm{A}}$ (given by large $\phi_{0}$ ) and therefore a smaller $\psi$, when $\tau_{\mathrm{G}}$ is constant. The other effect inferred from Figure 1 is linked to the growth rate $g_{R}$. The larger $g_{\mathrm{R}}$, the higher the likelihood of finding particles with a larger radius (cf. the size of the primary particles increase from the violet ones toward the red ones in Figure 1). Larger values of $g_{\mathrm{R}}$ lead to compact clusters and imply smaller $\tau_{\mathrm{G}}$, and larger $\psi$ values. Such an effect is more pronounced at smaller particles concentrations, for example, $\phi_{0}=1 \%$ versus $\phi_{0}=10 \%$. At smaller $\phi_{0}$, and at a fixed $g_{\mathrm{R}}$ (e.g., $\left.g_{\mathrm{R}}=1.00 \%\right)$, the aggregation characteristic time $\tau_{\mathrm{A}}$ increases (compared to that for situations where $\phi_{0}$ is larger) (cf. eq 13); however, the growth characteristic time $\tau_{\mathrm{G}}$ remains unaffected. In other words, growth becomes increasingly dominant when the aggregation rate decreases, as is best appreciated when considering the ratio of the two characteristic times, $\psi$ (cf. eq 15).

Summarizing, compact clusters are formed by a significant growth rate and by a larger occupied volume fraction. Therefore, $d_{\mathrm{m}}$ is expected to increase for both larger and smaller $\psi$ values. To appreciate the fractal nature of the clusters, the cluster mass $i$ is plotted against the hydrodynamic radius $R_{\mathrm{H}, i}$ for the cases $\phi_{0}=1 \%$ with $g_{\mathrm{R}}=0.00$ and $0.50 \%$ in Figure 2 .

In Figure 2, it can be seen that larger clusters remain on the continuous lines. Therefore, biases from the selected number of particles can be excluded for the range of cluster masses selected, namely, $10 \leq i \leq 5000$. From these plots and linearizing eq 1 , the mass-mobility exponent $d_{\mathrm{m}}$ and the fractal prefactor were computed. Figure 2 allows qualitative insights into the relevance of the growth process on the cluster mass distribution and the structural properties of the clusters to be gained. At comparable $\tau_{\mathrm{N}}$, larger clusters are formed if growth is present (cf. Figure 2c,d with a,b). Moreover, a larger degree of scatter is present in the data when growth occurs, especially at 

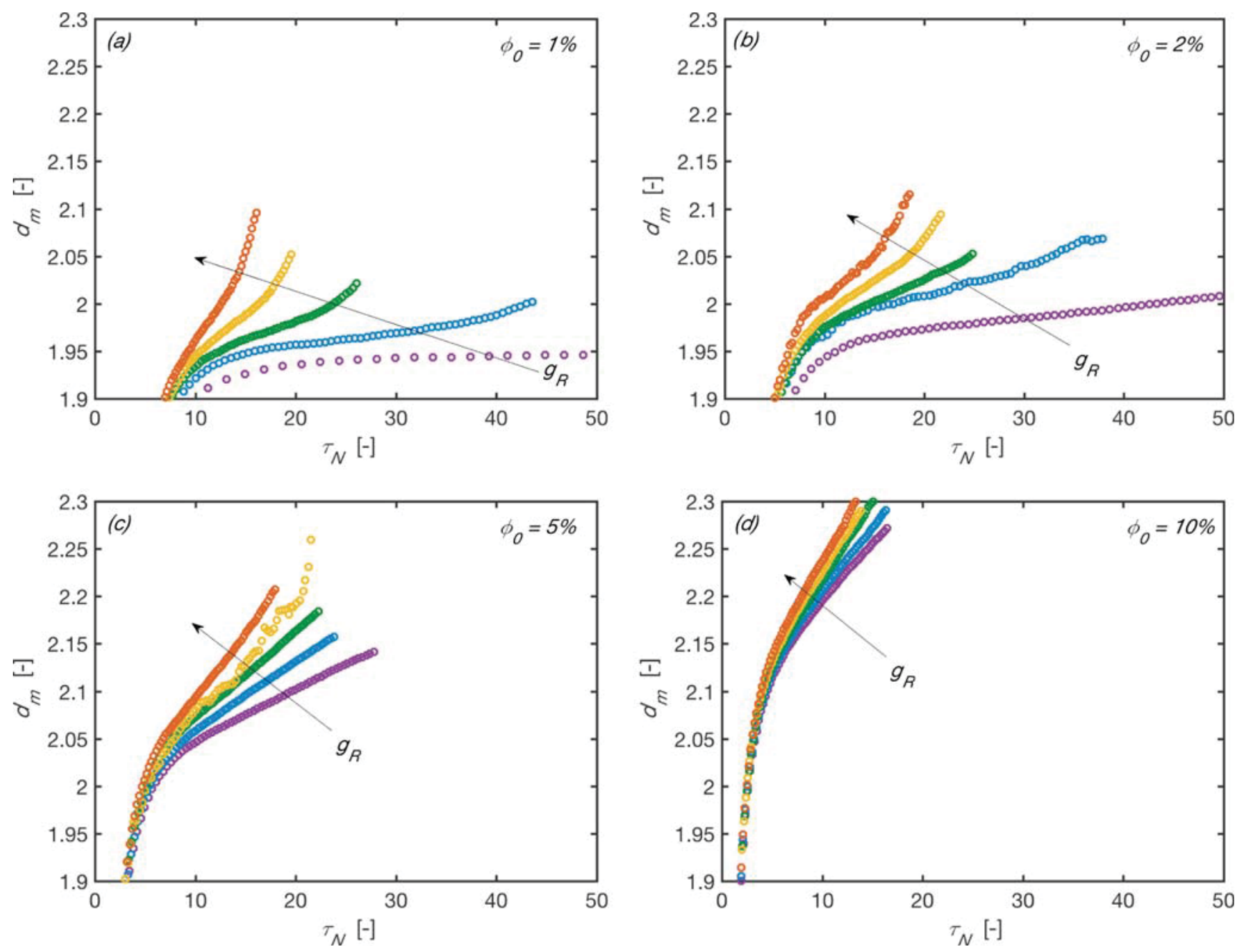

Figure 3. $d_{\mathrm{m}}$ vs $\tau_{\mathrm{N}}$ in DLCA at different $\phi_{0}$ and $g_{\mathrm{R}}$. (a) $\phi_{0}=1 \%$, (b) $\phi_{0}=2 \%$, (c) $\phi_{0}=5 \%$, and (d) $\phi_{0}=10 \%$. Color code: violet, $g_{\mathrm{R}}=0.00 \%$; blue, $g_{\mathrm{R}}$ $=0.25 \%$; green, $g_{\mathrm{R}}=0.50 \%$; yellow, $g_{\mathrm{R}}=0.75 \%$; red, $g_{\mathrm{R}}=1.00 \%$.

smaller sizes. This is due to the fact that growth introduces a further degree of variability in the cluster structure, by modifying the size of single particles belonging to a cluster. Such a difference is less significant for larger clusters, as the impact of single particle growth affects a large cluster less than a small one.

For a more quantitative assessment of the role of $\psi$ on the clusters' compactness, the mass-mobility exponent $d_{\mathrm{m}}$ (estimated through eq 1 ), is reported against the normalized time $\tau_{\mathrm{N}}$ in Figure 3 .

Note that no error bars are reported in Figure 3 because the standard error computed was found to be negligible (around $0.3 \%)$. The trends of $d_{\mathrm{m}}$ versus $\tau_{\mathrm{N}}$ in Figure 3 confirm the qualitative observations made when inspecting Figure 1 . The larger the initial occupied volume fraction, the larger the compactness (i.e., the larger $d_{\mathrm{m}}$ ) of the clusters (cf. Figure 3a and d). Also, the effect of the growth rate is confirmed: the larger the $g_{\mathrm{R}}$, the more compact the formed clusters become, as indicated by the arrows in Figure $3 a-d$. This latter effect becomes more pronounced for lower $\phi_{0}$. For instance, the $d_{\mathrm{m}}$ versus $\tau_{\mathrm{N}}$ set of curves at $\phi_{0}=10 \%$ exhibits similar trends in shape and absolute values, as compared to those of the $\phi_{0}=1 \%$ case. In other words, an increase of $g_{\mathrm{R}}$ has a more pronounced effect on the results, as $\phi_{0}$ decreases.
Notably, as the growth of the different particles occurs continuously throughout the simulation, the $d_{\mathrm{m}}$ steadily increases over time. Such an increase is also observed for the zero growth case (cf. violet curves in Figure 3). This can be rationalized recalling the aforementioned discrepancies arising at high concentration as compared to those at the ideal diluted DLCA regime. ${ }^{33-36,12}$ Notably, the $d_{\mathrm{m}}$ values observed (well above 2.0) significantly exceed the ones reported for ideal DLCA aggregation, which lie around 1.8. ${ }^{37}$ This is in agreement with existing modeling studies, which reported larger $d_{\mathrm{f}}$ values at large $\phi_{0} \cdot{ }^{33,34}$ In particular, when computing the fractal dimension in the zero growth case at $\phi_{0}=1 \%$, we obtain a value of 1.906 , as opposed to 1.885 , proposed by González et $\mathrm{al}^{34}$ This slight discrepancy could be related to the fact that González et al. employed an on-lattice MC, as opposed to our off-lattice simulations.

If sufficient simulation time was given, the mass-mobility exponents would reach values of $d_{\mathrm{m}} \approx 3$, once the cluster concentration decreased to such an extent that aggregation could be neglected and growth dominates, that is, $\psi$ increases (cf. eq 15).

As the clusters' $d_{\mathrm{m}}$ changes over time, it is expected that the aggregation kinetics will be affected as well. ${ }^{11,38}$ To prove this latter point and quantify the extent of such variation as a function of the growth rate, the 0th order moment $\mu_{0}$ (cf. eq 8 ) 

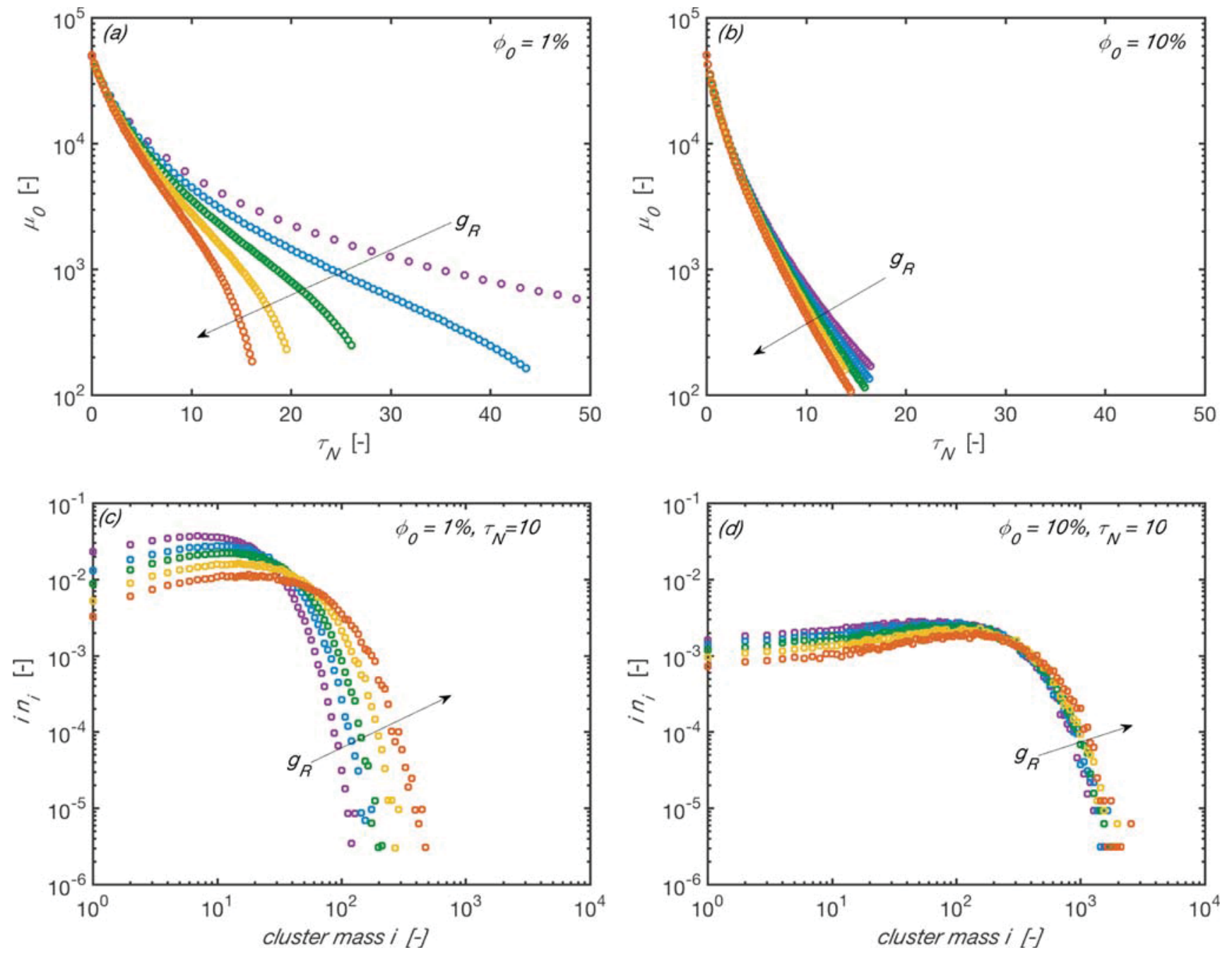

Figure 4. a, b) $\mu_{0}$ vs $\tau_{\mathrm{N}}$ in DLCA at different $\phi_{0}$ and $g_{\mathrm{R}}$. (a) $\phi_{0}=1 \%$, (b) $\phi_{0}=10 \%$. (c, d) DLCA distributions at $\tau_{\mathrm{N}}=10$ at different $\phi_{0}$ and $g_{\mathrm{R}}$. (c) $\phi_{0}=1 \%$, (d) $\phi_{0}=10 \%$. Color code: violet, $g_{\mathrm{R}}=0.00 \%$; blue, $g_{\mathrm{R}}=0.25 \%$; green, $g_{\mathrm{R}}=0.50 \%$; yellow, $g_{\mathrm{R}}=0.75 \%$; red, $g_{\mathrm{R}}=1.00 \%$.

is reported against $\tau_{\mathrm{N}}$ in Figure $4 \mathrm{a}, \mathrm{b}$ for the cases $\phi_{0}=1$ and $10 \%$.

Comparing Figure $4 a, b$, it is evident that increasing the initial occupied volume fraction (i.e., the particle concentration) speeds up the aggregation rate. ${ }^{35,36}$ This is due to the fact that aggregation is a second-order event, whose rate scales with the square of the concentration. This increase in aggregation rate is more pronounced for larger $g_{\mathrm{R}}$ as can be observed in both Figure $4 \mathrm{a}, \mathrm{b}$. This can be ascribed to the fact that the more the clusters grow, the larger the space they occupy and the larger the probability that they will further continue their aggregation. Notably, the influence of $g_{\mathrm{R}}$ is more significant at lower values of occupied fraction $\phi_{0}$, as can be seen by comparing the difference between the purple and the red curves (zero and maximum growth, respectively) in Figure $4 \mathrm{a}$ where $\phi_{0}=1 \%$, and Figure $4 \mathrm{~b}$, where $\phi_{0}=10 \%$. This is due to the fact that aggregation is dominating the rate of aggregation at the larger occupied volume fraction and growth cannot fully unravel its influence, as instead happens in the case at lower concentration (cf. Figure 4a).

Further insights into the impact of a change in $d_{\mathrm{m}}$ on the aggregation kinetics can be observed in Figure $4 c, d$, where the normalized cluster mass distributions, defined as $n_{i}=N_{i} / N$, are reported for $\phi_{0}=1$ and $10 \%$ at different growth rates in their weighted form $i n_{i}$.

Figure $4 c, d$ shows another facet of the aggregation kinetics in the presence of simultaneous growth and aggregation. The faster the clusters grow, the more space they occupy and the faster they will aggregate. As a result, a right-shift in the distributions is observed for faster growing clusters (cf. Figure 4c). Such an effect holds for all the occupied volume fractions under investigation, but its significance diminishes at larger $\phi_{0}$ (cf. Figure $4 \mathrm{~d}$ ). This can be explained recalling how the impact of growth on cluster compactness is particularly significant at a lower occupied volume fraction, as already discussed when introducing Figure 3 and the ratio of growth and aggregation characteristic time, $\psi$ (cf. eq 15).

Note that a larger $g_{\mathrm{R}}$ also implies clusters with a larger $d_{\mathrm{m}}$ (cf. Figure 3). This result might look counterintuitive, given that clusters consisting of the same number of particles agglomerate faster the more open they are, that is, the lower their $d_{\mathrm{m}}$. However, in the present case, the size of the primary particles building up the clusters increases with $g_{R}$. As a result, the clusters will have, at the same time, a larger degree of compactness and a larger spatial extension, agglomerating, thus, with other clusters with a larger probability, as compared to the nongrowing clusters. Therefore, the growth process indirectly 


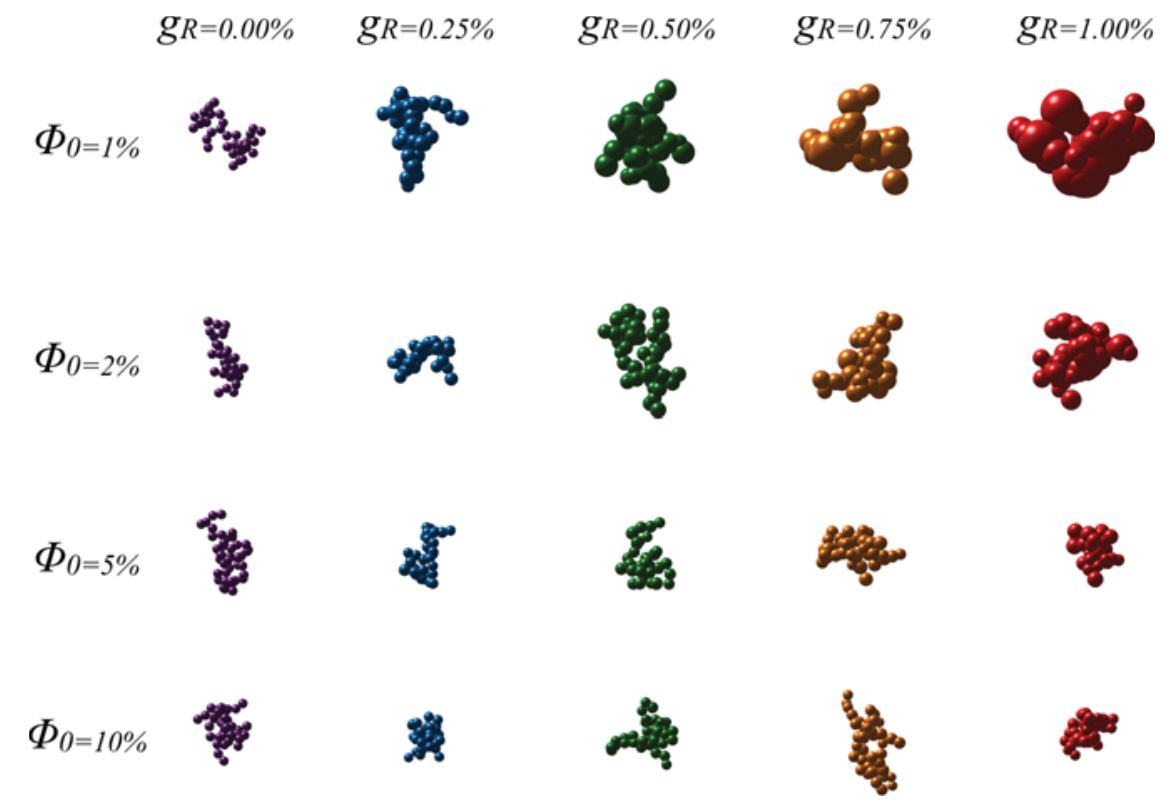

Figure 5. Examples of RLCA clusters retrieved at $\tau_{\mathrm{N}}=2$, with mass $i$ between $30<i<50$. Clusters are generated at different initial occupied volume fractions $\left(\phi_{0}=1,2,5\right.$, and $\left.10 \%\right)$ and growth rates $g_{\mathrm{R}}$. Color code: violet, $g_{\mathrm{R}}=0.00 \%$; blue, $g_{\mathrm{R}}=0.25 \%$; green, $g_{\mathrm{R}}=0.50 \%$; orange, $g_{\mathrm{R}}=0.75 \%$; red, $g_{\mathrm{R}}$ $=1.00 \%$.
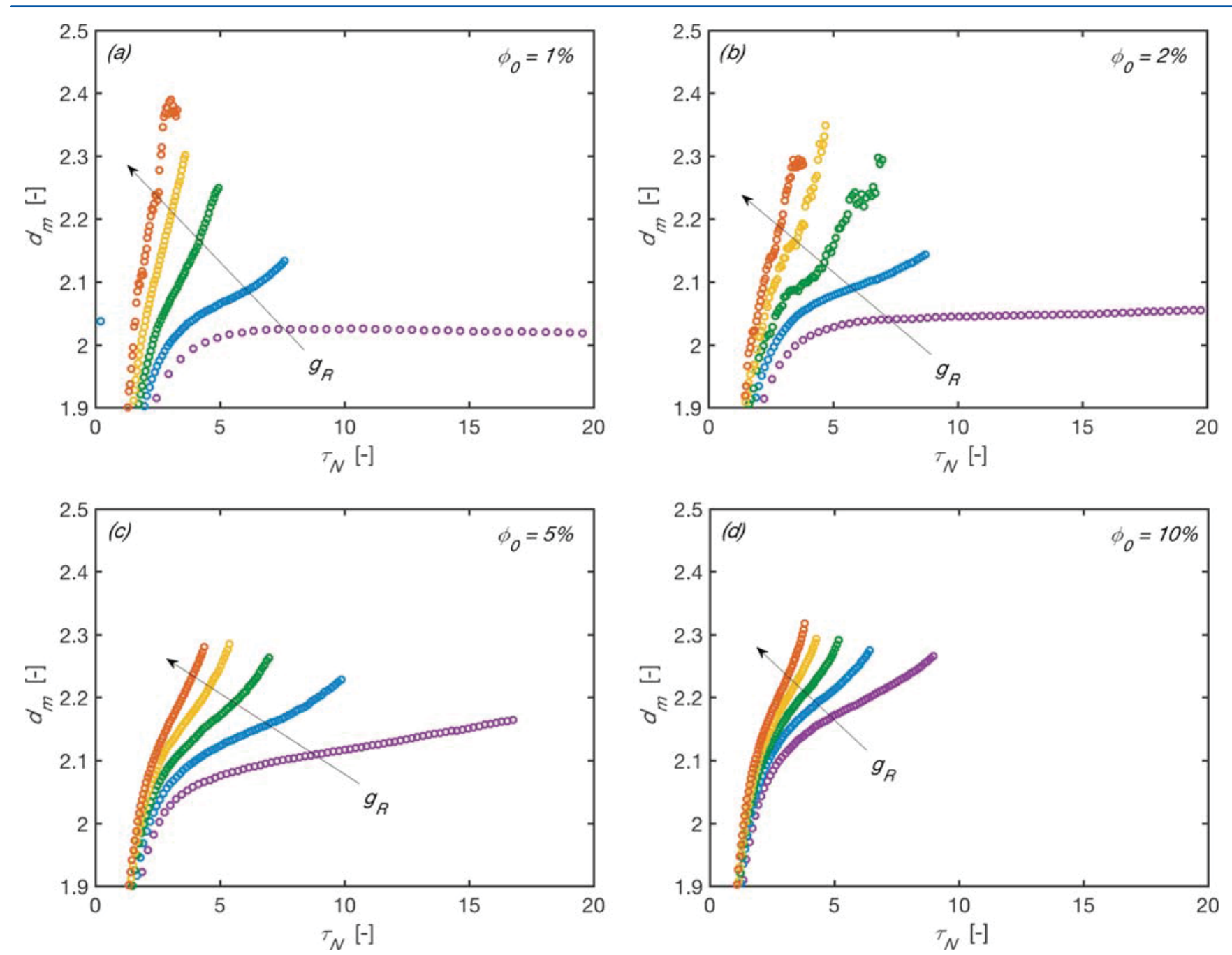

Figure 6. $d_{\mathrm{m}}$ vs $\tau_{\mathrm{N}}$ in RLCA with $p_{\mathrm{S}}=0.1$ at different $\phi_{0}$ and $g_{\mathrm{R}}$. (a) $\phi_{0}=1 \%$, (b) $\phi_{0}=2 \%$, (c) $\phi_{0}=5 \%$, (d) $\phi_{0}=10 \%$. Color code: violet, $g_{\mathrm{R}}=$ $0.00 \%$; blue, $g_{\mathrm{R}}=0.25 \%$; green, $g_{\mathrm{R}}=0.50 \%$; yellow, $g_{\mathrm{R}}=0.75 \%$; red, $g_{\mathrm{R}}=1.00 \%$. 

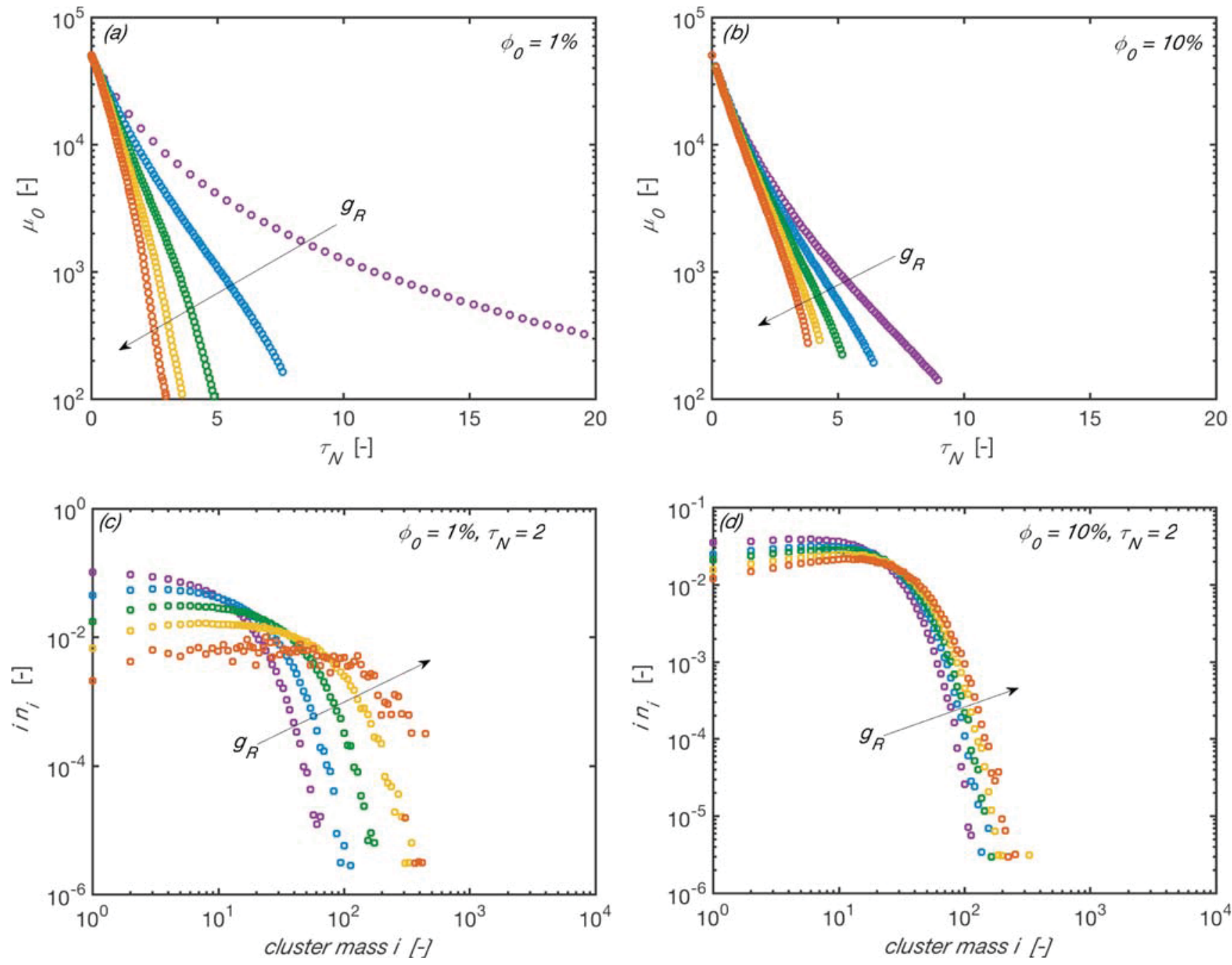

Figure 7. (a, b) $\mu_{0}$ vs $\tau_{N}$ in RLCA with $P_{\mathrm{S}}=0.1$ at different $\phi_{0}$ and $g_{\mathrm{R}}$. (a) $\phi_{0}=1 \%$, (b) $\phi_{0}=10 \%$; (c, d) RLCA distributions with $P_{\mathrm{S}}=0.1$ at $\tau_{\mathrm{N}}=2$ at different $\phi_{0}$ and $g_{\mathrm{R}}$. (c) $\phi_{0}=1 \%$, (d) $\phi_{0}=10 \%$. Color code: violet, $g_{\mathrm{R}}=0.00 \%$; blue, $g_{\mathrm{R}}=0.25 \%$; green, $g_{\mathrm{R}}=0.50 \%$; yellow, $g_{\mathrm{R}}=0.75 \%$; red, $g_{\mathrm{R}}=$ $1.00 \%$.

affects the agglomerating one, through the modification of the clusters' structure.

Further average quantities against $\tau_{\mathrm{N}}$ were computed for the explored set of $g_{\mathrm{R}}$ and $\phi_{0}$, such as the occupied volume fraction $\phi$, the particle volume fraction $\phi_{\mathrm{P}}$, and the average number of particles per cluster, $N_{\mathrm{AVE}}=\mu_{1} / \mu_{0}$. For the sake of brevity, these results are reported in the Supporting Information (cf. Figures S2-S4). The observed trends can be rationalized along the same principles discussed in the frame of the cluster concentration $\mu_{0}$ versus $\tau_{\mathrm{N}}$.

3.2. RLCA. In RLCA, the aggregation between clusters occurs only with a certain likelihood, the aforementioned sticking probability, $P_{S}$. In this set of simulations, $P_{\mathrm{S}}$ was set to equal 0.1 (cf. Table 1). Due to the lower value of $P_{\mathrm{S}}$ in RLCA as compared to that in the DLCA case (where $P_{S}=1$ ), the resulting clusters are typically more compact in RLCA. In fact, clusters tend to interpenetrate more with one another before aggregation occurs in RLCA, leading to the observed larger $d_{\mathrm{m}}{ }^{11,39}$ The degree of compactness of the clusters, or their $d_{\mathrm{m}}$, is also affected in this aggregation regime by the interplay of the characteristic times of aggregation and growth, or rather by their ratio, $\psi$ (cf. eq 15). To appreciate the cluster structures observed in this case, agglomerates at time $\tau_{\mathrm{N}}=2$ with masses between $30<i<50$ are reported in Figure 5 .

As in the DLCA case (cf. Figure 1), a pattern emerges. Clusters formed under concentrated conditions (large $\phi_{0}$ ) are denser (higher $d_{\mathrm{m}}$ ) compared to the ones formed under dilute conditions (cf. clusters in first and fourth row in Figure 5). This also happens when the particles do not grow (cf. violet clusters in Figure 5), as increasing the initial particle concentration is known to cause clusters to become denser. ${ }^{33,34,40}$ Moreover, as observed already in the DLCA case, increasing $g_{\mathrm{R}}$ causes the clusters to become more compact (cf. red clusters vs violet or blue clusters in Figure 5). Once more, this effect is more pronounced at low $\phi_{0}$, as the growth process dominates over the aggregation one for large values of $g_{\mathrm{R}}$ and low values of the product $P_{S} \phi_{0}$ (cf. eq 15). Despite the similarities between the clusters observed in DLCA (cf. Figure 1) and RLCA (cf. Figure 5 ), it is expected that the clusters' fractal dimensions are larger in RLCA. This effect is known to happen at zero growth ${ }^{11,38}$ and should be present when primary particles grow. Notably, such a higher degree of compactness can be rationalized by considering the definition of $\psi$ (cf. eq 15). In particular, the sticking probability $P_{\mathrm{S}}$ at the denominator is 1 in the DLCA case but equals 0.1 in the RLCA case. Therefore, as the 

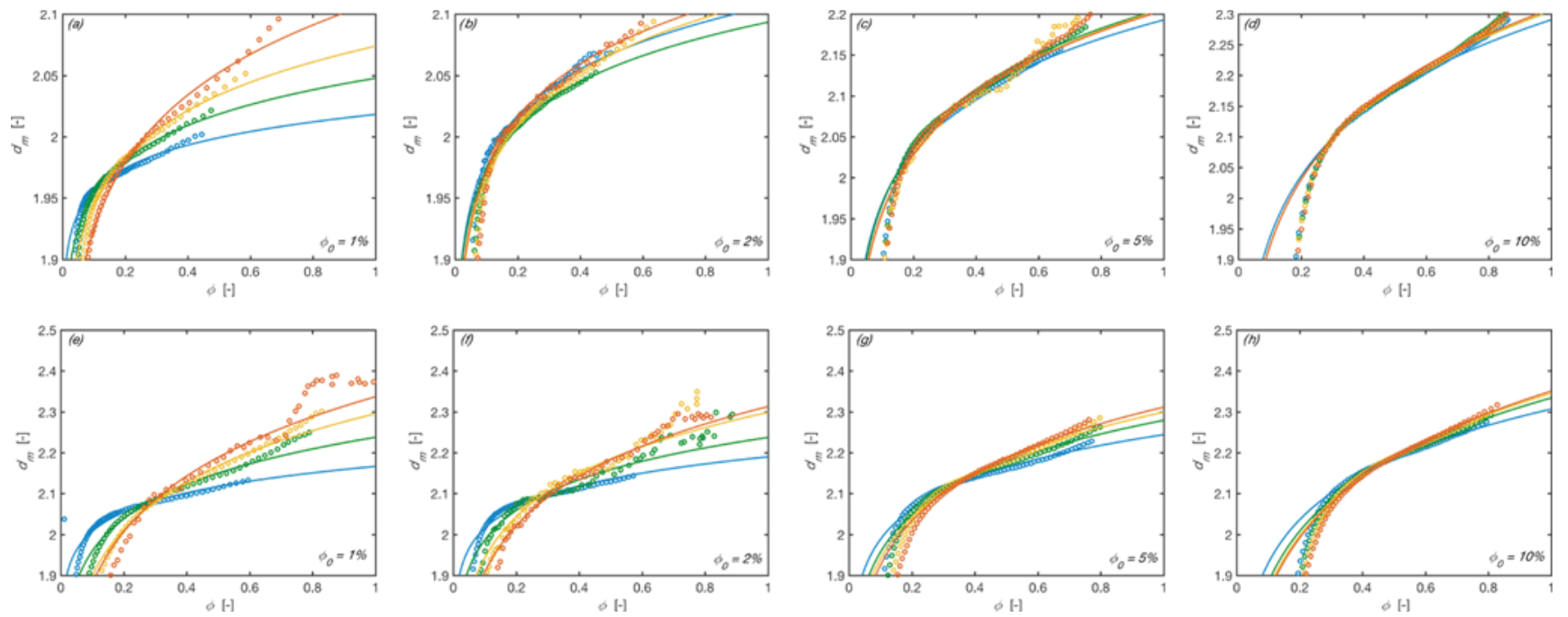

Figure 8. $d_{\mathrm{m}}$ values against $\phi$ in DLCA $(\mathrm{a}-\mathrm{d})$ and RLCA $(\mathrm{e}-\mathrm{h})$ with $P_{\mathrm{S}}=0.1$ at different $\phi_{0}$ and $g_{\mathrm{R}}$. $(\mathrm{a}, \mathrm{e}) \phi_{0}=1 \% ;(\mathrm{b}, \mathrm{f}) \phi_{0}=2 \% ;(\mathrm{c}, \mathrm{g}) \phi_{0}=5 \%$; $(\mathrm{d}, \mathrm{h}): \phi_{0}=10 \%$. Color code: blue, $g_{\mathrm{R}}=0.25 \%$; green, $g_{\mathrm{R}}=0.50 \%$; yellow, $g_{\mathrm{R}}=0.75 \%$; red, $g_{\mathrm{R}}=1.00 \%$. Circles represent the MC simulations; the continuous lines are fits using the correlation $d_{\mathrm{m}}=\kappa \phi^{\gamma}$.

remaining parameters defining $\psi$ (namely, $\phi_{0}$ and $g_{\mathrm{R}}$ ) were varied in the same range for the two simulation sets (cf. Table $1)$, it is expected that the explored $\psi$ values are larger in the RLCA case. Larger values of $\psi$ mean a more dominant role of growth over aggregation, which leads to larger values of $d_{\mathrm{m}}$, or a higher degree of compactness. A quantitative perspective of this reasoning is offered in Figure 6, where $d_{\mathrm{m}}$ is reported against $\tau_{\mathrm{N}}$ in RLCA conditions for the different parameter values explored.

Note that no error bars were reported in Figure 6 because the standard error computed was found to be negligible (around $0.3 \%$ ). Although the trends reported in Figure 6 are very similar to the corresponding ones in DLCA conditions (cf. Figure 3), it can be seen that larger values of $d_{\mathrm{m}}$ are reached in the RLCA case. It should be noticed that the typical $d_{\mathrm{m}}$ value of RLCA aggregation $\left(d_{\mathrm{m}, \mathrm{RLCA}} \approx 2.1\right)$ is largely overshot, with values reaching almost $d_{\mathrm{m}} \approx 2.4$. The rate of increase is more pronounced for larger $g_{\mathrm{R}}$ and lower $\phi_{0}$, as previously discussed. In particular, the differences among a set of curves (at fixed $\phi_{0}$ ) are more pronounced for smaller $\phi_{0}$, as the growth process dominates with more ease in diluted conditions. When the concentration of particles increases beyond a certain point, the role of growth gets increasingly small and little difference among the results is observed. In this respect, it is interesting to compare Figures $3 \mathrm{~d}$ and $6 \mathrm{~d}$, both reporting the case of high concentration aggregation, $\phi_{0}=10 \%$, for the same set of $g_{\mathrm{R}}$, but with different sticking probabilities. It can be seen that the difference among the curves is more pronounced in the RLCA case (cf. Figure 6d) than that in the DLCA case (Figure 3d). This can be rationalized as follows. As the sticking probability is smaller in the RLCA case (0.1 vs 1.0 in DLCA), the aggregation takes a longer time, which allows the growth process to still play a role, even at such a high particle concentration. As a result, different $g_{\mathrm{R}}$ values significantly impact the average cluster compactness in the RLCA case at high $\phi_{0}$, whereas they play less of a role in the DLCA case.

To appreciate the impact of the fractal dimension, and of the interplay between growth and aggregation, on the kinetics in the RLCA case, the cluster concentration $\mu_{0}$ is reported in Figure $7 \mathrm{a}, \mathrm{b}$.

Figure $7 \mathrm{a}, \mathrm{b}$ shows how the cluster concentration decreases faster at higher concentration (at fixed $g_{\mathrm{R}}$ ) and at larger growth (at fixed $\phi_{0}$ ). The same observation can be made when considering the normalized cluster mass distributions in the RLCA case, as shown in Figure 7c,d. These observations are analogous to those described for the DLCA case (cf. Figure 4) and will therefore not be discussed in further depth. Further, average quantities of the cluster mass distribution, such as $\phi$, $\phi_{\mathrm{P}}$, and $N_{\mathrm{AVE}}=\mu_{1} / \mu_{0}$, were computed for the RLCA case and are reported in the Supporting Information for the sake of brevity (cf. Figures S5-S7).

3.3. $d_{\mathrm{m}}$ Evolution. In the previous sections it was shown that the $d_{\mathrm{m}}$ actually increases in time for both the DLCA and RLCA regimes, during the aggregation process (cf. Figures 3 and 6). Therefore, it would be desirable to find an expression quantifying the $d_{\mathrm{m}}$ time-evolution for the cases where both aggregation and growth occur simultaneously. Note that the case at zero growth is not being considered, as González et al. ${ }^{33,34}$ already proposed a correlation for $d_{\mathrm{m}}$ as a function of the initial occupied volume fraction, $\phi_{0}$.

Given the underlying approximations necessary to relate the MC time to the physical time (cf. Section 2.2), we decided to seek a correlation based on the occupied volume fraction, $\phi$ (cf. eq 9). Notably, the occupied volume fraction $\phi$ increases during the clustering process for two reasons: (i) growth occurs and the already present clusters "expand", occupying even more space, and (ii) clustering leads to the formation of porous structures, whose voids are inaccessible to the other clusters, thus increasing the available occupied volume. $\phi$ is, hence, a quantity affected by growth and aggregation throughout the clustering process. Figure 8 shows the trends of $d_{\mathrm{m}}$ against $\phi$ for both the DLCA and RLCA cases for $g_{R}>0$.

The trends of $d_{\mathrm{m}}$ against $\phi$ can be rationalized along the same lines as the other quantities previously discussed (cf. Sections 3.1 and 3.2). The curves with nonzero growth were fit employing the following correlation

$$
d_{\mathrm{m}}=\kappa \phi^{\gamma}
$$

where $\kappa$ and $\gamma$ are fitting parameters. Note that the $d_{\mathrm{m}}$ increases for both larger $\kappa$ and $\gamma$. The selected power law (cf. eq 18) was able to satisfactorily describe the trends both in the DLCA and the RLCA cases (cf. continuous lines in Figure 8). Note that the fit using eq 18 was performed considering only the central 

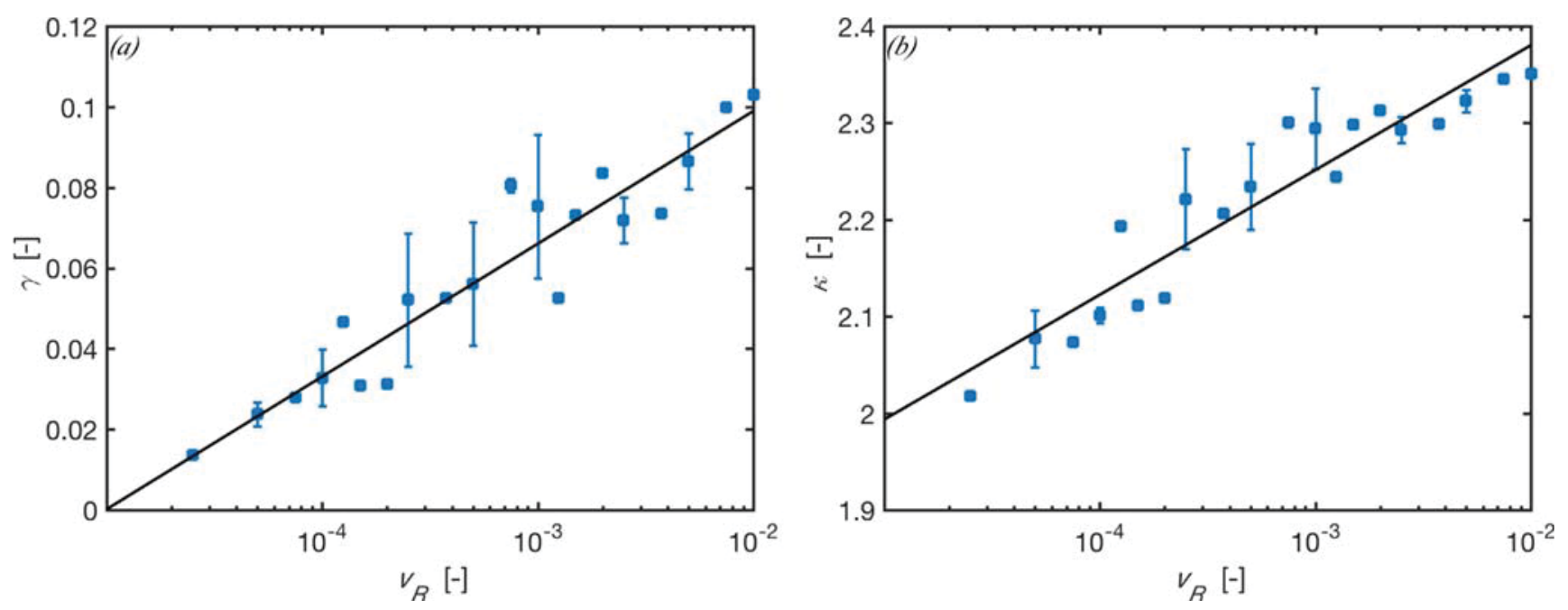

Figure 9. (a) Squares: fitted $\gamma$ (cf. eq 18) against $v_{\mathrm{R}}$ (cf. eq 21). The continuous line is a logarithmic function, where $\gamma=0.0143\left[\ln \left(v_{\mathrm{R}}\right)\right]+0.1648$, with $R^{2}=0.89$. (b) Fitted $\kappa$ (cf. eq 18) against $v_{\mathrm{R}}$ (cf. eq 21). The continuous line is a logarithmic function, where $\kappa=0.056\left[\ln \left(v_{\mathrm{R}}\right)\right]+2.6382$, with $R^{2}=0.89$. Whenever multiple $\gamma$ or $\kappa$ values were present for the same $v_{\mathrm{R}}$ value, an average thereof was obtained and an error bar was included.

portion of the data $d_{\mathrm{m}}$ versus $\phi$. The initial $d_{\mathrm{m}}$ increase was neglected, as it is due to the first cluster formation, where a proper $d_{\mathrm{m}}$ value cannot be strictly defined as too few "developed" clusters are present. The final part of the data $d_{\mathrm{m}}$ versus $\phi$ was neglected as the observed sharp $d_{\mathrm{m}}$ increase is ascribed to the incipient gelation. Considering those data points would bias the estimation of a scaling, accounting for phenomena which occur at a stage where the aggregation process is basically fully completed. Although the resulting fitting curves are reasonable (cf. Figure 8), it must be stressed that a different set of $\kappa$ and $\gamma$ was used to describe the evolution of each single $d_{\mathrm{m}}$ versus $\phi$ trend. However, it would be desirable to have a function predicting which values of $\kappa$ and $\gamma$ were to be used in each case, possibly as a function of the growth and aggregation parameters. To find an appropriate scaling law, one could attempt to write a differential equation quantifying the fractal-dimension evolution as a function of the occupied volume fraction where

$$
\frac{\mathrm{d} d_{m}}{\mathrm{~d} \phi}=\frac{\mathrm{d} d_{m}}{\mathrm{~d} t} \frac{\mathrm{d} t}{\mathrm{~d} \phi}
$$

Note that $\mathrm{d} t / \mathrm{d} \phi$ can be obtained by deriving the definition of occupied volume fraction (cf. eq 9) and inverting it. Given that $\phi=\frac{4}{3} \pi \frac{1}{L^{3}} \sum_{i=1}^{N_{\mathrm{C}}} N_{i} R_{\mathrm{H}, i}^{3}$ is a function of the entire cluster mass distribution, $N_{i}$, which in turn changes, due to both aggregation and growth, it is clear that no simple expression can be derived for $\mathrm{d} \phi / \mathrm{d} t$. Focusing on $\mathrm{d} d_{\mathrm{m}} / \mathrm{d} t$ instead, and recalling that $d_{\mathrm{m}}$ is bounded between its initial value, $d_{\mathrm{m}, 0}$, and 3 , its maximum value, a simple expression could be employed, following similar examples already present in the literature ${ }^{16,17,41,42}$

$$
\frac{\mathrm{d} d_{\mathrm{m}}}{\mathrm{d} t}=\frac{1}{\tau_{\mathrm{R}}}\left(3-d_{\mathrm{m}}\right)
$$

where $\tau_{\mathrm{R}}$ represents the characteristic time of restructuring, which is, in the present case, affected by both aggregation and growth. Although no explicit expression is known for $\tau_{\mathrm{R}}$ one can infer at least some dependencies on growth and aggregation parameters, based on the literature and the results in the present work. In particular, the mass-mobility exponent is known to increase for (i) larger initial occupied volume fraction $\phi_{0}$, (ii) larger growth rates (see ref 18 and this work), and (iii) smaller sticking probabilities. ${ }^{41}$ On the basis of these results and hypothesizing a simple linear dependency on the abovementioned parameters, one could write

$$
\frac{1}{\tau_{\mathrm{R}}}=v_{\mathrm{R}} \propto \frac{g_{\mathrm{R}} \phi_{0}}{P_{\mathrm{S}}}
$$

where $v_{\mathrm{R}}$ is the rate of the restructuring (i.e., of $d_{\mathrm{m}}$ increase), defined as the inverse of the characteristic time of restructuring. Overall, although this analysis does not lead to an analytical expression of $\mathrm{d} d_{\mathrm{m}} / \mathrm{d} \phi$, it suggests that the fractal dimension should change, during the clustering process, as a function of the rate of restructuring, $v_{\mathrm{R}}$. Therefore, it is reasonable to expect that correlations in the form $\kappa=\kappa\left(v_{\mathrm{R}}\right)$ and $\gamma=\gamma\left(v_{\mathrm{R}}\right)$ allow generalization of the fitting function of eq 18 to describe the DLCA and RLCA evolution of $d_{\mathrm{m}}$ against $\phi$. As a matter of fact, it turns out that the fitted parameters $\gamma$ and $\kappa$ do scale with $v_{\mathrm{R}}$ as shown in Figure 9.

Figure 9 shows how both $\kappa$ and $\gamma$ increase with $v_{\mathrm{R}}$ in a logarithmic fashion with an $R^{2}=0.89$. Note that such an increase in prefactor and exponent with $v_{\mathrm{R}}$ implies a larger $d_{\mathrm{m}}$ and reflects the occurring physical processes. A larger $v_{R}$ implies, as suggested by its definition (cf. eq 21), a larger growth, a larger initial particle concentration, or a larger stability toward aggregation (i.e., a smaller sticking probability). As already seen in the literature ${ }^{18,33,41}$ and in this work, the larger these quantities, the more compact the resulting clusters and therefore the larger the values of $\kappa$ and $\gamma$.

The reported empirical dependencies among $\gamma$ and $v_{R}$ and among $\kappa$ and $v_{\mathrm{R}}$, allow relation of the mass-mobility exponent to the clustering process through relevant physical quantities of the process (cf. eq 21). Notably, the found dependencies could be used in population balance equation models describing the simultaneous occurrence of growth and aggregation.

3.4. Gel Time. Similar to what has been done in Section 3.3, it would be desirable to establish a relation between the gel time $\left(t_{\text {gel }}\right)$ and the growth and aggregation processes. The gelation of a suspension is related to its viscosity, which, in turn, is a function of the occupied volume fraction $\phi$, and it is known that $t_{\text {gel }}=t_{\text {gel }}(\phi)$. In particular, a larger occupied volume fraction speeds up the gelation of a colloid suspension. ${ }^{43,44}$ 
When analyzing the factors affecting the occupied volume fraction (cf. Section 3.3), to find an expression describing $\mathrm{d} \phi /$ $\mathrm{d} t$, a complex pattern emerged, suggesting that $\phi$ changes as a function of both growth and aggregation. The faster any of these two processes (for which $\tau_{\mathrm{G}} \propto g_{\mathrm{R}}^{-1}, \tau_{\mathrm{A}} \propto\left(P_{\mathrm{S}} \phi_{0}\right)^{-1}$ ) becomes, the faster the increase in occupied volume fraction (cf. Figures S2 and S4); therefore, a faster gelation is expected to occur. To test this hypothesis, the gel times of the DLCA simulations are plotted in Figure 10 against the product $\tau_{\mathrm{A}} \tau_{\mathrm{G}}$.

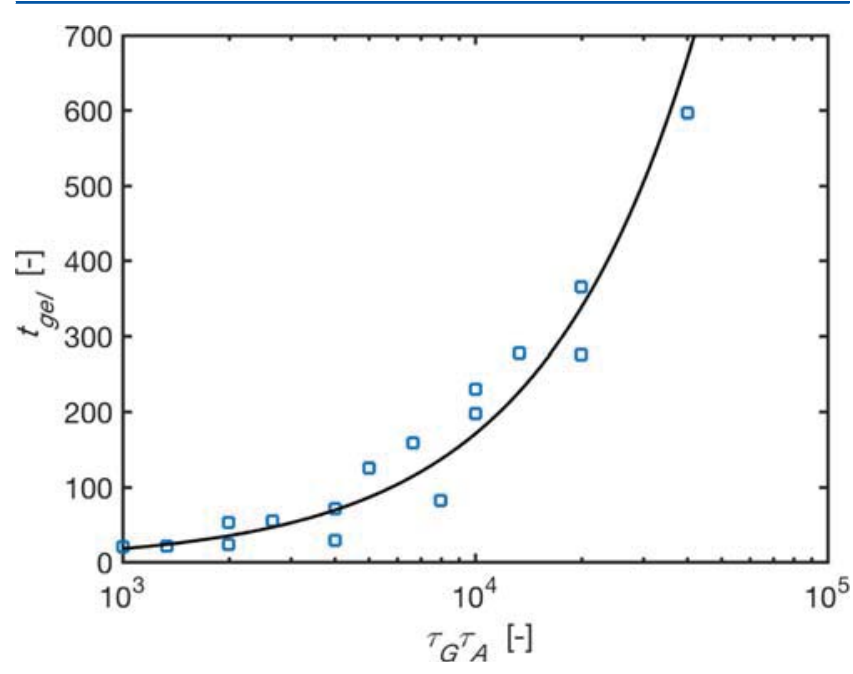

Figure 10. $t_{\mathrm{gel}}$ against $\tau_{\mathrm{A}} \tau_{\mathrm{G}}$ for the DLCA simulations (symbols). The continuous line represents a fitting curve in the form $t_{\mathrm{gel}}=$ $0.0192\left(\tau_{\mathrm{A}} \tau_{\mathrm{G}}\right)^{0.987}$, with $R^{2}=0.89$.

In Figure 10, it is possible to see how the gel time increases for both a slower growth rate (larger $\tau_{\mathrm{G}}$ ) and slower aggregation (larger $\tau_{\mathrm{A}}$ ). Note that only DLCA simulations were considered, given that no gelation was observed in the explored RLCA cases. Therefore, a full validation of the observed scaling $t_{\mathrm{gel}} \propto \tau_{\mathrm{A}} \tau_{\mathrm{G}}$ is not yet possible, but an indication for its validity exists. Fitting the gel times in the functional form $t_{\text {gel }}=a\left(\tau_{\mathrm{A}} \tau_{\mathrm{G}}\right)^{b}$ further corroborates the aforementioned correlation, cf. the continuous line in Figure 10. The retrieved exponent, $b=0.987$, is very close to 1 , with an $R^{2}=0.89$, indicating the reliability of the proposed scaling. Such scaling, that is, $t_{\text {gel }} \propto \tau_{\mathrm{A}} \tau_{\mathrm{G}}$, could be of help when trying to predict the gelation onset in systems where growth and aggregation occur simultaneously.

3.5. Impact on Aggregation Kinetics. The previously introduced correlation, $t_{\text {gel }} \propto \tau_{\mathrm{A}} \tau_{\mathrm{G}}$, stresses the impact of the growth and aggregation interplay on the aggregation kinetics. In fact, it was shown (cf. Figures 4 and 7 ) that in both the DLCA and RLCA regimes, the aggregation process is faster; the total concentration decreases faster and the cluster mass distributions $n_{i}$ are progressively right-shifted, at larger growth rates. Interestingly, when plotting the scaled distribution, $f_{i}$ is defined as $^{34}$

$$
f_{i}=\frac{n_{i}}{N_{0} W_{\mathrm{AVE}}^{2}}
$$

against $i / W_{\mathrm{AVE}}$, where $W_{\mathrm{AVE}}=\mu_{2} / \mu_{1}$ (cf. moments definition in eq 8 ), it turns out that the distributions overlap, revealing a selfpreserving character, as shown in Figure 11.

The same scaling has been used by González et al. ${ }^{34}$ to analyze the cluster mass distributions at different concentrations. The observed superimposition in Figure 11 suggests that although the aggregation rate is affected by the growth rate the same scaling applies, which means that from the point of view of cluster aggregation kinetics, the effect of growth has a similar effect to that of concentration. This is an additional confirmation of the behavior discussed in the previous sections of the work.

3.6. Overview of the Model Features. The cluster shapes that can be formed under the explored conditions of aggregation and growth are summarized in Figure 12.

Monodisperse particles can aggregate in the presence (cf. red clusters in Figure 12) or in the absence (cf. blue clusters in Figure 12) of growth phenomena. In dilute conditions, where $\phi_{0}=1 \%$, with a sticking probability $P_{S}=1$, open clusters, reflecting the DLCA aggregation mechanism, are obtained (Figure 12a). By reducing the sticking probability to $P_{\mathrm{S}}=0.1$, more compact clusters typical of the RLCA regime are formed (Figure 12b). Increasing the initial particle concentration to $\phi_{0}$ $=10 \%$ further increases the cluster compactness (Figure 12c). These very same observations hold also in the presence of
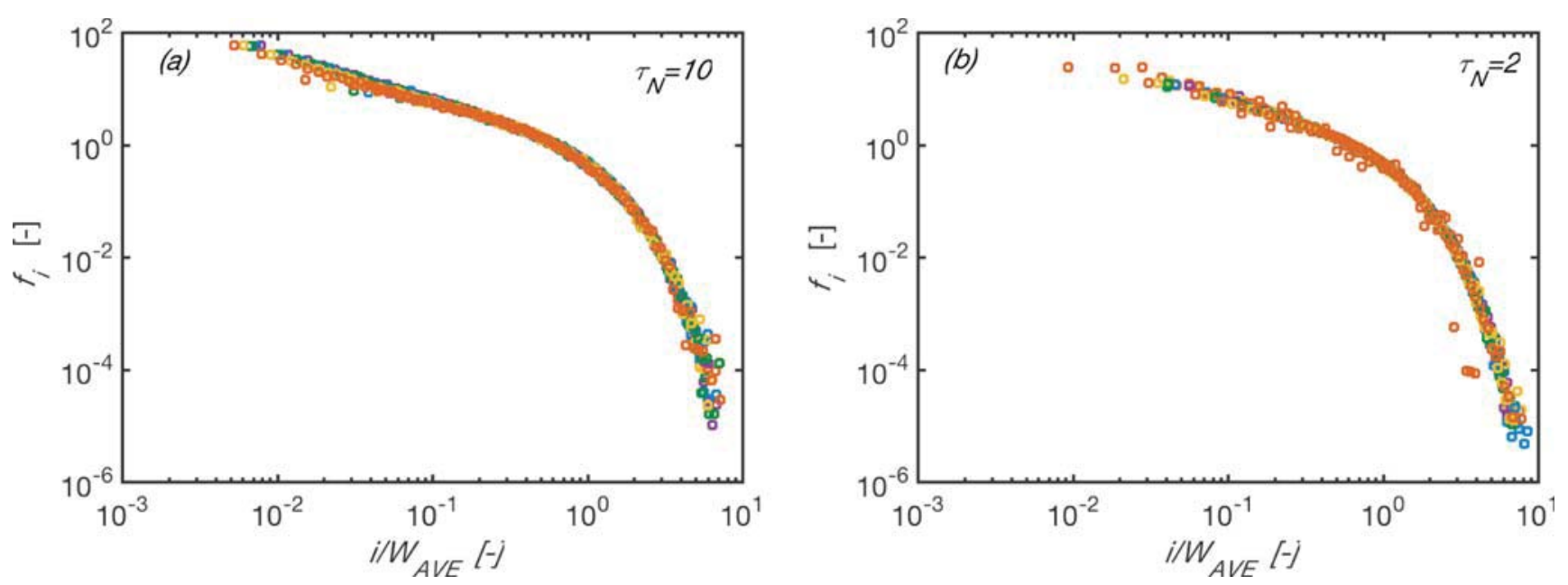

Figure 11. (a) Scaled DLCA distributions at $\tau_{N}=10$ for $\phi_{0}=1,10 \%$, (b) scaled RLCA distributions with $P_{S}=0.1$ at $\tau_{N}=2$ for $\phi_{0}=1,10 \%$. The differently colored symbols, partially overlapped, represent the different growth rates. Color code: violet, $g_{R}=0.00 \%$; blue, $g_{R}=0.25 \%$; green, $g_{R}=$ $0.50 \%$; yellow, $g_{\mathrm{R}}=0.75 \%$; red, $g_{\mathrm{R}}=1.00 \%$. 


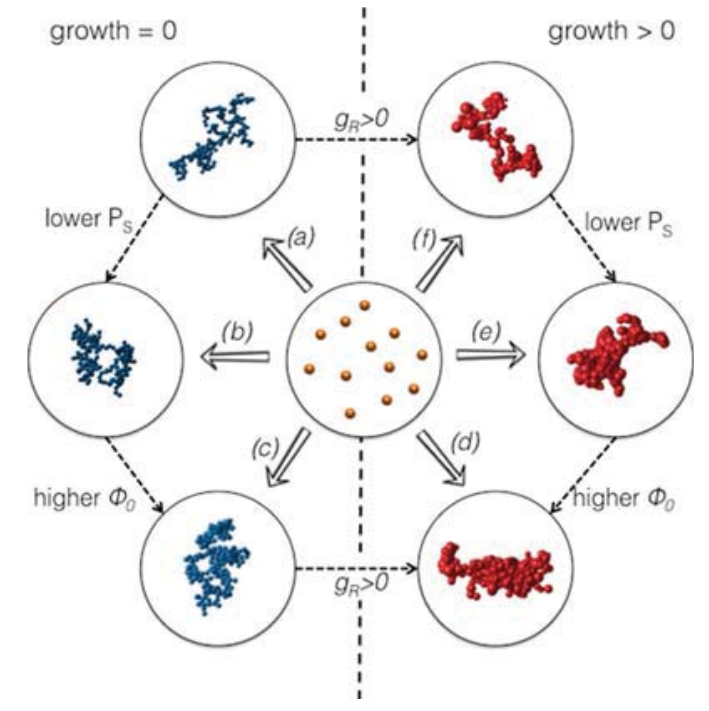

Figure 12. Overview of the structures that can be obtained by the MC model. Blue clusters are obtained by aggregation only $\left(g_{\mathrm{R}}=0 \%\right)$, whereas red clusters are obtained by aggregation and growth $\left(g_{\mathrm{R}}=\right.$ $1 \%)$. (a, f) DLCA with $\phi_{0}=1 \%$; (b, e) RLCA with $\phi_{0}=1 \%$ and $P_{\mathrm{S}}=$ $0.1 ;(c, d)$ RLCA with $\phi_{0}=10 \%$ and $P_{S}=0.1$.

growth (Figure 12d-f). Once more, it can be appreciated how the aggregating and growing clusters present a larger $d_{\mathrm{m}}$ as compared to that of their nongrowing counterparts. Growth has, indeed, a strong reshaping effect.

\section{CONCLUSIONS}

In the present work, an off-lattice MC model accounting for simultaneous particle growth and aggregation was developed. By varying the growth rate $\left(g_{\mathrm{R}}\right)$, the initial occupied volume fraction $\left(\phi_{0}\right)$, and the cluster sticking probability $\left(P_{S}\right)$, the model features were explored in the DLCA and RLCA regimes.

It turned out that the mass-mobility exponent $d_{\mathrm{m}}$ increases during the clustering process for (i) larger values of the occupied volume fraction $\phi_{0}$, (ii) larger growth rates $g_{\mathrm{R}}$ and (iii) lower sticking probabilities $P_{\mathrm{S}}$ (cf. Figures 3 and 6). These findings can be rationalized as follows. A large occupied volume fraction implies that clusters agglomerate with each other not only through their "tips", as they do in dilute conditions, but also through their cores, therefore forming denser structures. Large growth rates, $g_{\mathrm{R}}$, fill the "voids" of aggregating clusters, and lower sticking probabilities allow clusters to rearrange and interpenetrate more before aggregation occurs, again increasing $d_{\mathrm{m}}$.

To quantify these findings, a correlation relating $d_{\mathrm{m}}$ to the occupied volume of the system, in the form of $d_{\mathrm{m}}=\kappa \phi^{\gamma}$, was shown to effectively describe the evolution of the fractal dimension during the clustering process (cf. Figure 8). The fitting parameters $\kappa$ and $\gamma$ were proven to scale with the rate of change of the fractal dimension, $v_{\mathrm{R}} \propto g_{\mathrm{R}} \phi_{0} / P_{\mathrm{S}}$, and correlations in the form $\kappa=\kappa\left(v_{\mathrm{R}}\right)$ and $\gamma=\gamma\left(v_{\mathrm{R}}\right)$ were proposed to generalize the relation $d_{\mathrm{m}}=\kappa \phi^{\gamma}$ (cf. Figure 9). The obtained correlation could be employed in population balance equations based models studying growth and aggregation, possibly improving their kinetic predictions.

In fact, the impact of the fractal dimension on the aggregation kinetics could be appreciated from the present MC study. Notably, increasing initial occupied volume fractions and growth rates resulted in a faster decrease of the total cluster population, and a right-shift in the cluster mass distribution (cf. Figures 4 and 7). These results were observed for both DLCA and RLCA simulations. Moreover, it was shown that growth affects time-evolution in a similar manner to an increase in concentration. Cluster mass distributions obtained at different growth rates overlap when using a scaling developed for aggregation under concentrated conditions, revealing the typical self-preserving nature of DLCA and RLCA mechanisms (cf. Figure 11).

Interestingly, a correlation between the gel time and the characteristic times of growth $\left(\tau_{\mathrm{G}}\right)$ and aggregation $\left(\tau_{\mathrm{A}}\right)$ was found in the DLCA case, suggesting that $t_{\text {gel }} \propto \tau_{\mathrm{A}} \tau_{\mathrm{G}}$ (cf. Figure 10). The latter correlation could be of help when trying to predict gelation and clogging onset in reactors and crystallizers.

Despite the underlying simplifying assumptions (e.g., constant growth over time), the provided MC model, along with the proposed correlations, could be of help in the development of a universal agglomeration kernel in crystallization, where typically both growth and agglomeration occur.

\section{ASSOCIATED CONTENT}

\section{S Supporting Information}

The Supporting Information is available free of charge on the ACS Publications website at DOI: 10.1021/acs.jpcb.6b12682.

Calculation of the hydrodynamic radius of a cluster made of interpenetrating particles having different sizes; convergence of $d_{\mathrm{m}}$ with $N_{\mathrm{MAX}}$; kinetic information, further graphs $\left(\phi, \phi_{\mathrm{P}}, N_{\mathrm{AVE}}\right.$ against $\left.\tau_{\mathrm{N}}\right)$ for the DLCA and RLCA case (PDF)

\section{AUTHOR INFORMATION}

\section{Corresponding Authors}

*E-mail: slazzari@mit.edu (S.L.).

*E-mail: marco.lattuada@unifr.ch (M.L.).

\section{ORCID}

Stefano Lazzari: 0000-0002-6688-1467

\section{Notes}

The authors declare no competing financial interest.

\section{ACKNOWLEDGMENTS}

Both S.L. and M.L. gratefully acknowledge the Swiss National Science Foundation (SNSF) for financial support (S.L. grant numbers P2EZP2_159128 and P300P2_167683, M.L. grant number PP00P2_159258).

\section{REFERENCES}

(1) Ochsenbein, D. R.; Schorsch, S.; Vetter, T.; Mazzotti, M.; Morari, M. Growth Rate Estimation of $\beta$ l-Glutamic Acid from Online Measurements of Multidimensional Particle Size Distributions and Concentration. Ind. Eng. Chem. Res. 2014, 53, 9136-9148.

(2) van Embden, J.; Chesman, A. S. R.; Jasieniak, J. J. The Heat-Up Synthesis of Colloidal Nanocrystals. Chem. Mater. 2015, 27, 22462285.

(3) Kashchiev, D. Nucleation: Basic Theory With Applications; Butterworth Heinemann: Oxford, 2000; p 529.

(4) Chen, J.; Sarma, B.; Evans, J. M. B.; Myerson, A. S. Pharmaceutical Crystallization. Cryst. Growth Des. 2011, 11, 887-895.

(5) Lee, A. Y.; Erdemir, D.; Myerson, A. S. Crystal Polymorphism in Chemical Process Development. Annu. Rev. Chem. Biomol. Eng. 2011, 2, 259-280.

(6) Mura, F.; Zaccone, A. Effects of shear flow on phase nucleation and crystallization. Phys. Rev. E 2016, 93, No. 042803. 
(7) Salvalaglio, M.; Vetter, T.; Giberti, F.; Mazzotti, M.; Parrinello, M. Uncovering Molecular Details of Urea Crystal Growth in the Presence of Additives. J. Am. Chem. Soc. 2012, 134, 17221-17233.

(8) Marchisio, D. L.; Barresi, A. A.; Garbero, M. Nucleation, growth, and agglomeration in barium sulfate turbulent precipitation. AIChE J. 2002, 48, 2039-2050.

(9) Schöll, J.; Lindenberg, C.; Vicum, L.; Brozio, J.; Mazzotti, M. Precipitation of alpha L-glutamic acid: determination of growth kinetics. Faraday Discuss. 2007, 136, 247-264.

(10) Lindenberg, C.; Scholl, J.; Vicum, L.; Mazzotti, M.; Brozio, J. Lglutamic acid precipitation: Agglomeration effects. Cryst. Growth Des. 2008, 8, 224-237.

(11) Friedlander, S. K. Smoke, Dust, and Haze Fundamentals of Aerosol Dynamics, 2nd ed.; Oxford University Press: New York, 2000; p 407.

(12) Lazzari, S.; Nicoud, L.; Jaquet, B.; Lattuada, M.; Morbidelli, M. Fractal-like structures in colloid science. Adv. Colloid Interface Sci. 2016, 235, 1-13.

(13) Weitz, D. A.; Huang, J. S.; Lin, M. Y.; Sung, J. Limits of the fractal dimension for irreversible kinetic aggregation of gold colloids. Phys. Rev. Lett. 1985, 54, 1416-1419.

(14) Chakraborti, R. K.; Gardner, K. H.; Atkinson, J. F.; Van Benschoten, J. E. Changes in fractal dimension during aggregation. Water Res. 2003, 37, 873-883.

(15) Kostoglou, M.; Konstandopoulos, A. G. Evolution of aggregate size and fractal dimension during Brownian coagulation. J. Aerosol Sci. 2001, 32, 1399-1420.

(16) Eggersdorfer, M. L.; Kadau, D.; Herrmann, H. J.; Pratsinis, S. E. Multiparticle Sintering Dynamics: From Fractal-Like Aggregates to Compact Structures. Langmuir 2011, 27, 6358-6367.

(17) Lazzari, S.; Jaquet, B.; Colonna, L.; Storti, G.; Lattuada, M.; Morbidelli, M. Interplay between Aggregation and Coalescence of Polymeric Particles: Experimental and Modeling Insights. Langmuir 2015, 31, 9296-9305.

(18) Baldyga, J.; Jasinska, M.; Orciuch, W. Barium sulphate agglomeration in a pipe - An experimental study and CFD modeling. Chem. Eng. Technol. 2003, 26, 334-340.

(19) Besenhard, M. O.; Chaudhury, A.; Vetter, T.; Ramachandran, R.; Khinast, J. G. Evaluation of Parameter Estimation Methods for Crystallization Processes Modeled via Population Balance Equations. Chem. Eng. Res. Des. 2015, 94, 275-289.

(20) Hartman, R. L.; Naber, J. R.; Zaborenko, N.; Buchwald, S. L.; Jensen, K. F. Overcoming the Challenges of Solid Bridging and Constriction during Pd-Catalyzed $\mathrm{C}-\mathrm{N}$ Bond Formation in Microreactors. Org. Process Res. Dev. 2010, 14, 1347-1357.

(21) Hartman, R. L. Managing Solids in Microreactors for the Upstream Continuous Processing of Fine Chemicals. Org. Process Res. Dev. 2012, 16, 870-887.

(22) Thanh, N. T. K.; Maclean, N.; Mahiddine, S. Mechanisms of Nucleation and Growth of Nanoparticles in Solution. Chem. Rev. 2014, $114,7610-7630$.

(23) Coppens, M. O.; Froment, G. F. Diffusion and Reaction in a Fractal Catalyst Pore .1. Geometrical Aspects. Chem. Eng. Sci. 1995, 50, $1013-1026$.

(24) Sorensen, C. M. Light Scattering by Fractal Aggregates: A Review. Aerosol Sci. Technol. 2001, 35, 648-687.

(25) Meakin, P. Models for Colloidal Aggregation. Annu. Rev. Phys. Chem. 1988, 39, 237-267.

(26) Meakin, P. Formation of Fractal Clusters and Networks by Irreversible Diffusion-Limited Aggregation. Phys. Rev. Lett. 1983, 51, $1119-1122$

(27) Lattuada, M.; Wu, H.; Morbidelli, M. A simple model for the structure of fractal aggregates. J. Colloid Interface Sci. 2003, 268, 106120.

(28) Lattuada, M.; Wu, H.; Morbidelli, M. Radial density distribution of fractal clusters. Chem. Eng. Sci. 2004, 59, 4401-4413.

(29) Weitz, D. A.; Oliveria, M. Fractal Structures Formed by Kinetic Aggregation of Aqueous Gold Colloids. Phys. Rev. Lett. 1984, 52, $1433-1436$.
(30) Busa, J.; Dzurina, J.; Hayryan, E.; Hayryan, S.; Hu, C. K.; Plavka, J.; Pokorny, I.; Skrivanek, J.; Wu, M. C. ARVO: A Fortran package for computing the solvent accessible surface area and the excluded volume of overlapping spheres via analytic equations. Comput. Phys. Commun. 2005, 165, 59-96.

(31) Kowacz, M.; Putnis, C.; Putnis, A. The effect of cation: anion ratio in solution on the mechanism of barite growth at constant supersaturation: Role of the desolvation process on the growth kinetics. Geochim. Cosmochim. Acta 2007, 71, 5168-5179.

(32) Spanos, N.; Koutsoukos, P. G. Kinetics of precipitation of calcium carbonate in alkaline $\mathrm{pH}$ at constant supersaturation. Spontaneous and seeded growth. J. Phys. Chem. B 1998, 102, 66796684.

(33) González, A. E.; Martínez-López, F.; Moncho-Jordá, A.; Hidalgo-Alvarez, R. Two-dimensional colloidal aggregation: Concentration effects. J. Colloid Interface Sci. 2002, 246, 227-234.

(34) González, A. E.; Martínez-López, F.; Moncho-Jordá, A.; Hidalgo-Alvarez, R. Concentration effects on two- and threedimensional colloidal aggregation. Physica A 2002, 314, 235-245.

(35) Heine, M. C.; Pratsinis, S. E. Brownian coagulation at high concentration. Langmuir 2007, 23, 9882-9890.

(36) Lattuada, M. Predictive Model for Diffusion-Limited Aggregation Kinetics of Nanocolloids under High Concentration. J. Phys. Chem. B 2012, 116, 120-129.

(37) Lin, M. Y.; Lindsay, H. M.; Weitz, D. A.; Klein, R.; Ball, R. C.; Meakin, P. Universal Diffusion-Limited Colloid Aggregation. J. Phys.: Condens. Matter 1990, 2, 3093-3113.

(38) Lin, M. Y.; Lindsay, H. M.; Weitz, D. A.; Ball, R. C.; Klein, R.; Meakin, P. Universality in Colloid Aggregation. Nature 1989, 339, 360-362.

(39) Lin, M. Y.; Lindsay, H. M.; Weitz, D. A.; Ball, R. C.; Klein, R.; Meakin, P. Universal reaction-limited colloid aggregation. Phys. Rev. A 1990, 41, 2005-2020.

(40) Nicoud, L.; Lattuada, M.; Yates, A.; Morbidelli, M. Impact of aggregate formation on the viscosity of protein solutions. Soft Matter 2015, 11, 5513-5522.

(41) Kim, A. Y.; Berg, J. C. Fractal aggregation: Scaling of fractal dimension with stability ratio. Langmuir 2000, 16, 2101-2104.

(42) Gruy, F. Modelling of aggregate restructuring in a weakly turbulent flow (vol 395, pg 54, 2012). Colloids Surf., A 2012, 403, 169.

(43) Lazzari, S.; Maggioni, G. M.; Soos, M.; Wu, H.; Morbidelli, M. Shear-stability and gelation of inverse latexes. Soft Matter 2013, 9, 10866-10876.

(44) Zaccone, A.; Gentili, D.; Wu, H.; Morbidelli, M. Shear-induced reaction-limited aggregation kinetics of Brownian particles at arbitrary concentrations. J. Chem. Phys. 2010, 132, No. 134903. 